\title{
Mathematische Modellierungskompetenz fördern durch Lösungsplan oder Dynamische Geometrie-Software? Empirische Ergebnisse aus dem LIMo-Projekt
}

\author{
Corinna Hankeln (D) - Gilbert Greefrath (D)
}

Eingegangen: 20. Mai 2020 / Angenommen: 7. November 2020 / Online publiziert: 27. November 2020 (C) Der/die Autor(en) 2020

Zusammenfassung Mathematisches Modellieren ist inzwischen ein fester Bestandteil des deutschen Mathematik-Unterrichts. Es existieren unterschiedliche Ansätze, wie der Aufbau dieser Kompetenz unterstützt werden kann. Der vorliegende Artikel stellt die Ergebnisse einer quantitativen Studie vor, bei der die Auswirkungen des Einsatzes zweier unterschiedlicher Hilfsmittel, zum einen eines strategischen Instruments, dem sogenannten Lösungsplan, und zum anderen einer dynamischen Geometrie-Software, auf den Aufbau von Modellierungskompetenz untersucht wurden. Dazu werden zunächst die theoretisch angenommene Existenz unterschiedlicher Teilkompetenzen, bestehend aus Vereinfachen, Mathematisieren, Interpretieren und Validieren, auch empirisch mit Hilfe eines Rasch-Modells nachgewiesen und anschließend eine Intervention in 44 nordrhein-westfälischen Klassen ausgewertet, bei der die Lernenden entweder mit einem der beiden Hilfsmittel oder mit herkömmlichen Mitteln an Modellierungsaufgaben arbeiteten. Es zeigen sich nur geringe Unterschiede zwischen den Gruppen, allerdings scheinen sich die eingesetzten Hilfsmittel unterschiedlich effektiv für verschiedene Teilkompetenzen zu erweisen. Mögliche Ursachen sowie methodische Einschränkungen werden diskutiert.

Schlüsselwörter Modellieren · Lösungsplan · Dynamische Geometrie-Software · LIMo · Teilkompetenzen 


\title{
Fostering Mathematical Modelling Competency via Solution Plan or Dynamic Geometry Software? Empirical Results of the LIMo-Project
}

\begin{abstract}
Mathematical modelling is by now a binding part of German mathematics education. There are several approaches how to promote the development of this competency. This article presents the results of a quantitative study which analyses the effects of two different devices, a strategic instrument called « solution plan » and a dynamic geometry software, on the development of modelling competency. To do so, the theoretically assumed existence of different modelling sub-competencies is empirically shown with the help of the Item-Response-Theory. Secondly, the authors evaluate an intervention in 44 North-Rhine Westphalian classes, during which students worked on modelling task with either one of the devices or with conventional tools. Even though there were only slight differences between the three groups, the two devices seem to be differently effective for each sub-competency. Possible reasons as well as methodological flaws are discussed.
\end{abstract}

Keywords Mathematical modelling - Solution plan - Dynamic geometry software · LIMo $\cdot$ Subcompetency

\section{Einführung}

In den letzten Jahrzehnten wurde das Potenzial der Integration mathematischen Modellierens in den Mathematikunterricht umfassend untersucht. Weltweit wird mathematisches Modellieren immer stärker in Standards und den Mathematikunterricht integriert (Frejd 2011; Kaiser et al. 2015) und nimmt in der internationalen mathematikdidaktischen Diskussion einen hohen Stellenwert ein (Kaiser und Sriraman 2006). Zu möglichen sinnvollen Unterstützungsmaßnahmen beim Lernen mathematischen Modellierens gibt es verschiedene Ansätze. So kann etwa das Wissen über den Modellierungskreislauf (Galbraith und Clatworthy 1990; Tanner und Jones 1995), das zur Verfügung stellen heuristischer Lösungsbeispiele (Zöttl et al. 2010) oder das Nutzen eines strategischen Lösungsplans (Schukajlow et al. 2015a) Modellierungsprozesse unterstützen und fördern. Im Detail kann auch das Erstellen mathematischer und ggf. auch situativer Skizzen (Rellensmann et al. 2017) sowie die Aufforderung multiple Lösungen zu erstellen (Schukajlow et al. 2019) zu einer Steigerung der Modellierungskompetenz beitragen. In diesem Beitrag stellen wir die Auswirkungen des Einsatzes eines strategischen Lösungsplans und des digitalen Werkzeugs Dynamische Geometrie-Software (DGS) auf die Entwicklung von Modellierungskompetenz vor. 


\section{Mathematisches Modellieren}

\subsection{Begriff des mathematischen Modellierens}

Mit mathematischem Modellieren wird nach Pollak (1977, S. 255 ff.) ein bestimmter Aspekt der angewandten Mathematik bezeichnet, der als mehrmaliges Durchlaufen eines Modellierungskreislaufs beschrieben werden kann. Mathematische Modellierungsprozesse umfassen die wichtigen Teilprozesse des Übersetzens der ,Situation, die modelliert werden soll, in mathematische Begriffe, Strukturen und Relationen“ (KMK 2004, S. 8) sowie Ergebnisse in ,der entsprechenden Situation [zu] interpretieren und [zu] prüfen“ (KMK 2004, S. 8). Ein ausführliches Modell des Modellierens, das von Blum und Leiß $(2005,2007)$ entwickelt wurde, beinhaltet u. a. ein aus der Erforschung des Textverständnisses (Reusser 1997) motiviertes Situationsmodell. Darin wird der Prozess des Individuums, welches das Modell erstellt, von der Ausgangssituation zu deren mentaler Repräsentation als erster Schritt dargestellt. Anschließend wird das Problem zunächst auf der Sachebene vereinfacht. Dies führt zu einem Modell in der Realität, welches man häufig mit Realmodell bezeichnet. Nun folgt der Schritt in die Mathematik, zum mathematischen Modell. Mit Hilfe dieses Modells wird dann eine mathematische Lösung ermittelt, die schließlich wieder auf das reale Problem bezogen werden muss (s. Abb. 1). Zu verschiedenen Zwecken können unterschiedlich detaillierte Modellierungskreisläufe verwendet werden. Der Modellierungskreislauf von Blum und Leiß $(2005,2007)$ beschreibt die verschiedenen Teilprozesse des mathematischen Modellierens wie das Verstehen, Vereinfachen, etc. (s. Abb. 1) detaillierter als viele andere Modellierungskreisläufe (Borromeo Ferri 2006). Daher verwenden wir diesen Kreislauf für unsere weiteren Überlegungen.

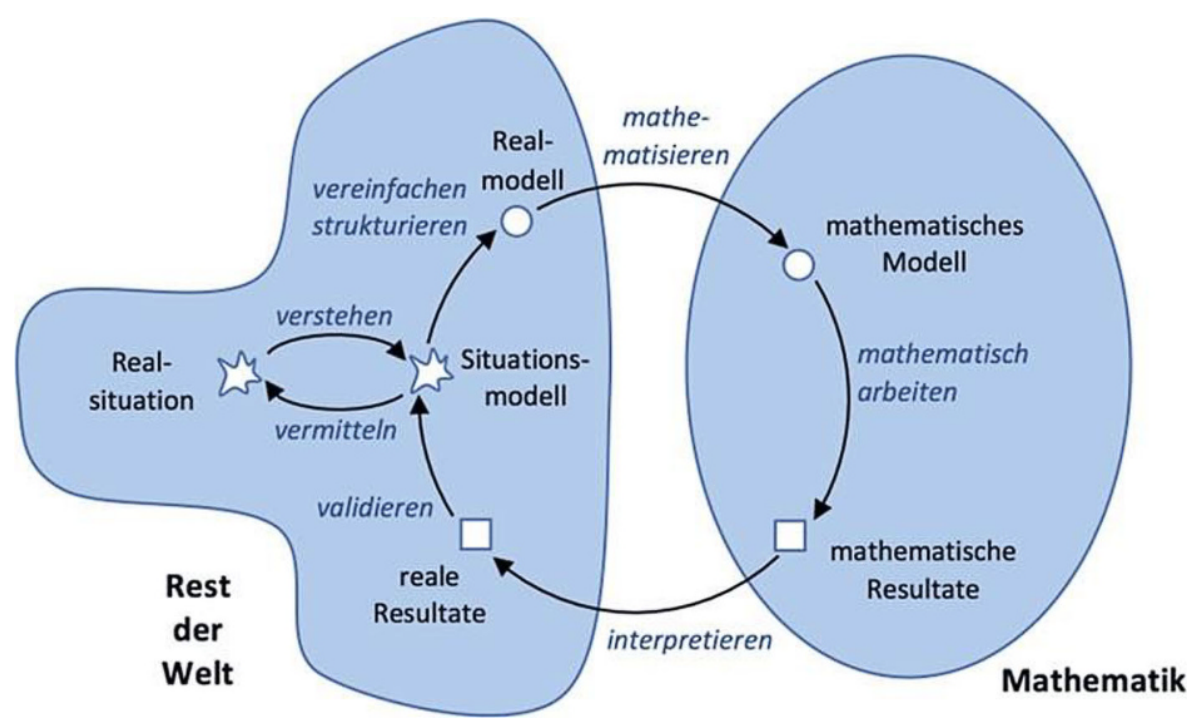

Abb. 1 Modellierungskreislauf von Blum und Leiß (2005, S. 19, 2007, S. 225) 


\subsection{Modellierungskompetenz}

Unter Modellierungskompetenz verstehen wir die Fähigkeit, ein Problem in einer gegebenen Situation der realen Welt zu identifizieren, dieses in die Mathematik zu übersetzen und die Lösung des entsprechenden mathematischen Problems in Bezug auf die gegebene Situation zu interpretieren und zu validieren (Niss et al. 2007, S. 12). Dies beschränkt sich nicht auf Fähigkeiten und Fertigkeiten, sondern umfasst auch ihren reflektierten Einsatz im Leben und die Bereitschaft, diese Fähigkeiten und Fertigkeiten selbstständig zu nutzen (Blomhøj und Jensen 2007; Maaß 2006).

Die Fähigkeit, einen Teilprozess im Modellierungskreislauf (vgl. Abb. 1) durchzuführen, kann als eine spezifische Teilkompetenz des mathematischen Modellierens angesehen werden, während Modellierungskompetenz sich auf Fähigkeiten bezieht, den gesamten Modellierungsprozess durchzuführen und über ihn zu reflektieren (Kaiser 2007; Maaß 2004, 2006). Im Folgenden haben wir detaillierte Beschreibungen aufgeführt, die die Art der Teilkompetenzen verdeutlichen sollen (Greefrath et al. 2013, S. 19; Мaaß 2006, S. 116f.):

- Verstehen: Die Schülerinnen und Schüler konstruieren ein eigenes mentales Modell zu einer gegebenen Problemsituation und verstehen so die Fragestellung.

- Vereinfachen: Schülerinnen und Schüler machen auf die Situation bezogen Annahmen, erkennen beeinflussende Größen, stellen Beziehungen zwischen den Größen her und suchen nach relevanten Informationen.

- Mathematisieren: Schülerinnen und Schüler übertragen die relevanten Größen und Beziehungen ggf. vereinfacht in ein mathematisches Modell und wählen dazu eine geeignete mathematische Darstellungsform.

- Mathematisch arbeiten: Schülerinnen und Schüler wenden heuristische Strategien und mathematisches Wissen zur Lösung des mathematischen Problems an.

- Interpretieren: Schülerinnen und Schüler übersetzten die mathematischen Resultate in außermathematische Situationen, verallgemeinern für spezielle Situationen entwickelte Lösungen und stellen Problemlösungen angemessen sprachlich dar.

- Validieren: Schülerinnen und Schüler überprüfen und reflektieren gefundene Lösungen, revidieren ggf. Teile des Modells, falls Lösungen der Situationen nicht angemessen sind und überlegen, ob andere Lösungen oder Modelle möglich sind

- Vermitteln: Die Schülerinnen und Schüler beziehen die im Situationsmodell gefundenen Antworten auf die Realsituation und beantworten so die Fragestellung.

Verwendet man andere Kreislaufmodelle als Ausgangspunkt, so kann man auch anders akzentuierte Teilkompetenzen des Modellierens erhalten. Mathematisches Arbeiten wurde aus Gründen der Vollständigkeit in die Liste der Kompetenzen aufgenommen, da mathematisches Arbeiten auch ein Teilprozess im Modellierungskreislauf ist. Es ist jedoch anzumerken, dass mathematisches Arbeiten für Modellierungsprozesse nicht so typisch ist wie z.B. das Mathematisieren. Während das Mathematisieren bei den meisten anderen allgemeinen mathematischen Kompetenzen wie Problemlösen oder Beweisen keine Rolle spielt, ist es beim mathematischen Arbeiten anders. 


\subsection{Studien zur Erfassung von Modellierungskompetenz}

Modellierungskompetenz ist messbar, unterscheidet sich nachweislich von rein technisch-mathematischen Kompetenzen (Blum et al. 2004) und ist nicht so stark vom inhaltlichen Bereich abhängig (Harks et al. 2014). Ausgehend von unterschiedlichen Auffassungen der Modellierungskompetenz wurden unterschiedliche Formate zur Erfassung der Modellierungskompetenz eingesetzt. Beschränkt man sich auf die Erhebung mittels schriftlicher Tests, so lassen sich zwei Grundprinzipien der Aufgabengestaltung unterscheiden. Zum einen werden Aufgaben eingesetzt, die nur Teilprozesse des Modellierens abfragen (atomistisches Vorgehen) und zum anderen Aufgaben, die das komplette Durchlaufen eines Modellierungskreislaufes erfordern (holistisches Vorgehen) (Blomhøj und Jensen 2003). Beide Vorgehensweisen haben jeweils ihre Vor- und Nachteile: Wie bereits bei den obigen Erläuterungen deutlich wurde, besteht die Fähigkeit zu modellieren aus mehr, als nur aus der Summe der einzelnen Teilkompetenzen (Maaß 2006). Werden demnach nur Testaufgaben mit atomistischem Fokus eingesetzt, so kann keine Aussage darüber getroffen werden, ob eine Person dazu in der Lage ist, einen kompletten Modellierungskreislauf zu durchlaufen und die einzelnen Teilkompetenzen in sinnvoller Weise miteinander zu verknüpfen. Daher wurden in einigen Studien holistische Modellierungsaufgaben eingesetzt, die gemäß unterschiedlicher Bewertungsschemata verschieden detailliert ausgewertet werden (Kreckler 2015; Reit 2016; Rellensmann et al. 2017; Schukajlow et al. 2015b).

Ein Nachteil dieser Erhebungsweise ist, dass die Teilprozesse des Modellierens in einer Aufgabe aufeinander aufbauen und daher nicht unabhängig voneinander zu bewerten sind. Aus diesem Grund nutzen andere Studien atomistische Aufgaben, um ein möglichst breites Bild der verschiedenen Teilkompetenzen zu erhalten und werten die Summe dieser Aufgaben als allgemeine Modellierungskompetenz (Haines et al. 2001; Maaß 2004). Der Vorteil eines solchen Testformats liegt vor allem darin, dass kontextbezogene Stärken oder Schwächen der Schülerinnen und Schüler nicht so stark ins Gewicht fallen, da innerhalb einer relativ kurzen Zeit eine Vielzahl unterschiedlicher Kontexte und Aufgabenstellungen eingesetzt werden kann. Allerdings können durch die Summierung der einzelnen Ergebnisse keine spezifischen Aussagen über die Kompetenzen eines Lernenden in den verschiedenen Teilprozessen des Modellierens getroffen werden. Um zu untersuchen, ob sich die verschiedenen Teilkompetenzen des Modellierens auch empirisch als separate Leistungsdispositionen erfassen lassen, ist besonders der Rückgriff auf die probabilistische Testtheorie (Bond und Fox 2007) hilfreich, bei der explizit verschiedene theoretische Kompetenzmodelle auf ihre empirische Passung hin überprüft werden können. Wenn sich die qualitativ beobachteten verschiedenen Teilkompetenzen des Modellierens auch quantitativ empirisch fassen lassen, so ist dies eine weitere Untermauerung der theoretischen Überlegungen hinsichtlich der Teilkompetenzen. Erste Ansätze zur Untersuchung dieser Frage sind bei Zöttl (2010) und Brand (2014) zu finden, die sowohl atomistische als auch holistische Testaufgaben einsetzen und die erhobenen Daten mit Hilfe der probabilistischen Testtheorie auswerten. In beiden Fällen zeigt sich, dass sich verschiedene Teilkompetenzen des Modellierens durchaus auch empirisch trennen lassen. Allerdings wurde in diesen Arbeiten die ver- 
schiedenen Teilkompetenzen des Modellierens noch nicht einzeln betrachtet. Zöttl (2010) trennt drei Teilprozesse jeweils bestehend aus 1) Verstehen - Vereinfachen/ Strukturieren - Mathematisieren, 2) mathematisch Arbeiten und 3) Interpretieren Validieren. Brand (2014) nutzt ebenfalls diese Unterteilung, nimmt aber das Verstehen im ersten Teilprozess nicht gesondert auf. In keiner der beiden Arbeiten werden allerdings Modelle diskutiert, in denen auch die Teilkompetenzen Vereinfachen und Mathematisieren sowie Interpretieren und Validieren voneinander getrennt werden. Es besteht somit noch Forschungsbedarf, ob sich diese Kompetenzen mit Hilfe eines neu zu entwickelnden Testinstruments überhaupt empirisch voneinander unterscheiden lassen.

Über die Erhebung der Teilkompetenzen des Modellierens hinaus wird in verschiedenen Studien auf die Bedeutung von metakognitiven Kompetenzen im Rahmen von Modellierungsprozessen hingewiesen (Kaiser 2007; Stillman 2011; Vorhölter et al. 2019). So kann das Wissen über den Modellierungskreislauf Modellierungsprozesse positiv beeinflussen (Galbraith und Clatworthy 1990; Schukajlow et al. 2015a) und durch vermitteltes Metawissen Modellierungskompetenz gefördert werden (Zöttl et al. 2010). Insbesondere kann vermitteltes Strategiewissen Prozeduren in Modellierungsprozessen verbessern (Tropper 2019).

\section{Hilfsmittel beim mathematischen Modellieren}

Um diesem Forschungsdesiderat nachgehen zu können, muss zunächst geklärt werden, was genau die betrachteten Hilfsmittel Dynamische Geometrie-Software und Lösungsplan charakterisiert und welche empirischen Erkenntnisse bereits in Bezug auf ihren Einfluss auf Lernprozesse bereits vorliegen.

\subsection{Digitale Werkzeuge: Dynamische Geometrie Software}

\subsubsection{Begriffsklärung}

Im Folgenden betrachten wir eine dynamische Geometrie-Software (DGS) als spezielles digitales Werkzeug. Charakteristisch für eine solche Software, wie etwa das in der vorliegenden Studie genutzte Programm GeoGebra, sind zum einen, dass Konstruktionen, wie sie auch von Hand möglich sind, meist mit deutlich geringerem Aufwand durchführbar sind. Beispielsweise ist GeoGebra dazu in der Lage, bei einem Kreis, der durch drei Punkte konstruiert wurde, durch einen Mausklick den Mittelpunkt anzeigen zu lassen. Damit entfallen einige Hilfskonstruktionen per Hand. Weiterhin ist besonders die Dynamik des Programms zu betonen, insbesondere der sogenannte Zugmodus. Bei dieser Funktion können Objekte durch einen Mausklick ausgewählt und an eine beliebige Position gezogen werden. Objekte, die von dem bewegten Objekt abhängen, verändern sich dabei dynamisch mit. So können nicht nur Zeichnungen geometrischer Objekte erstellt werden, sondern auch die geometrischen Beziehungen zwischen den einzelnen Elementen konstruiert werden (Olive et al. 2009). Beispielsweise können Veränderungen im Zugmodus zeigen, ob ein geometrischer Zusammenhangs korrekt erstellt wurde (Meyer 2013). Dabei 
kann es sich um Verschiebungen oder Drehungen eines Objekts oder auch um die Veränderungen einzelner Objekte bei simultaner Kovariation der übrigen Bestandteile handeln. Eine solche Zuginvarianz bietet die Möglichkeit und kann es sogar erfordern, geometrische Gesetzmäßigkeiten, die ein Problem oder eine geometrische Objekt-Darstellung ausmachen, zu erkennen (Sacristán et al. 2009).

\subsubsection{Werkzeugnutzung beim Modellieren}

Die Nutzung digitaler Werkzeuge im Mathematikunterricht und insbesondere beim mathematischen Modellieren ist in den letzten Jahren immer mehr in das Zentrum des Interesses gerückt (Greefrath et al. 2018). Bereits seit Beginn der 2000er-Jahre wurden digitale Werkzeuge auch in verschiedene Kreislauf-Darstellungen des Modellierens integriert. Zu Beginn wurden kaum unterschiedliche Funktionen der Werkzeuge differenziert, sondern besonders die Möglichkeiten der Werkzeug-Nutzung bei dem Teilschritt des mathematischen Arbeitens gesehen. So etwa bei Galbraith et al. (2003), die Technologie als externen Faktor betrachten, der unterstützend beim Lösen auf mathematische Prozesse und Routinen einwirkt. Andere Autoren (Adan et al. 2005; Savelsbergh et al. 2008; Siller und Greefrath 2010) betonen insbesondere, dass durch die Werkzeugnutzung auch eine neue Art Computer-Modell Eingang in den Modellierungskreislauf findet. Gemäß dieser Auffassung muss ein reales oder mathematisches Modell zunächst in eine Art digitales Modell am Computer übertragen werden, bevor eine Lösung des Problems bestimmt werden kann. Dazu sind ebenfalls Fähigkeiten in der Bediensprache des jeweils verwendeten Werkzeugs notwendig (vgl. Greefrath und Mühlenfeld 2007). Eine dritte Betrachtungsweise stellt die vollständige Integration des Werkzeugs in den Modellierungskreislauf dar. Empirische Studien (Geiger 2011) weisen darauf hin, dass die digitalen Werkzeuge

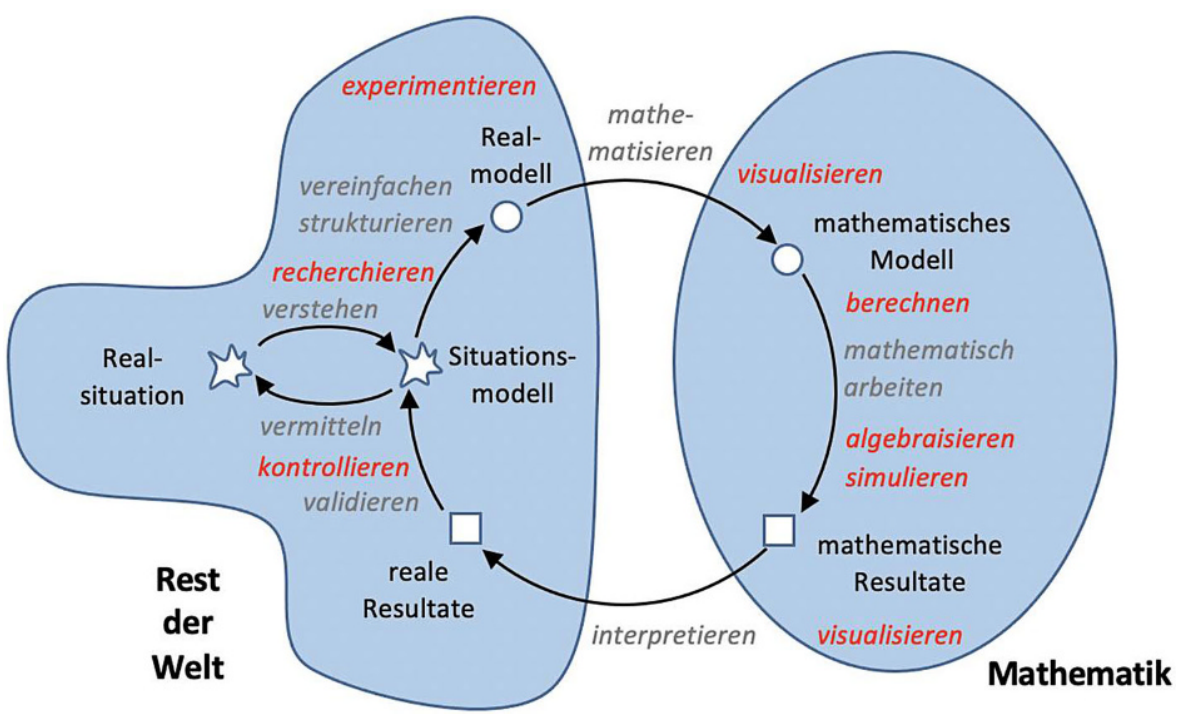

Abb. 2 Funktionen digitaler Werkzeuge beim Modellieren (farbig markiert) 
durchaus in fast allen Modellierungsschritten genutzt werden. Greefrath (2011) beispielsweise nennt dabei sieben verschiedene Funktionen digitaler Werkzeuge beim Modellieren (Recherchieren, Experimentieren, Visualisieren, Simulieren, Algebraisieren, Berechnen und Kontrollieren) und verortet diese im siebenschrittigen Modellierungskreislauf nach Blum und Leiß (2005, 2007; vgl. Abb. 2).

Diese Überlegungen zur Verwendung einer DGS beim Modellieren wurden für die Nutzung einer DGS konkretisiert (Hankeln 2019). Es zeigt sich in theoretischen Überlegungen wie auch in empirischen Untersuchungen (Hertleif und Greefrath 2016), dass die DGS in den unterschiedlichen Teilschritten des Modellierens jeweils mehrere Funktionen erfüllen kann, welche auf unterschiedliche Arten realisiert werden können. Insbesondere der Einfluss der Werkzeugleiste, die den Lernenden vor Augen führt, über welche Möglichkeiten sie noch verfügen in ihrer Modellkonstruktion, wirkt als anregend für die Metakognition über das eigene Vorgehen.

\subsubsection{Empirische Befunde zur DGS-Nutzung beim Modellieren}

Auch wenn die Nutzung digitaler Werkzeuge beim Modellieren immer stärker in den Fokus der Forschung treten, gibt es kaum Studien zu deren Wirksamkeit beim Modellieren. Insbesondere quantitative Daten liegen kaum vor, allerdings gibt es einige Studien, die die Verwendung einer DGS beim Modellieren aus qualitativer Sicht näher beleuchten. So beobachteten und befragten Christou et al. (2005) sechs Lehramtsstudierende beim Bearbeiten einer Modellierungsaufgabe. Dort nutzen die Probandinnen und Probanden die DGS zum Verstehen des Problems, zur Exploration, zur Visualisierung sowie zur Kontrolle. In einer zweijährigen Langzeitstudie fand Mousoulides (2011) anhand der Bearbeitungsprozesse von 21 vierzehnjährigen Schülerinnen und Schüler, die an Modellierungsaufgaben arbeiteten, dass ,the capabilites of dynamic tools, such as GeoGebra, can positively influence students' explorations and understandings in complex problem solving“ (Mousoulides 2011, S. 117). Dabei benutzten die beobachteten Schülerinnen und Schüler eine Vielzahl an in der DGS implementierten Werkzeugen, die ihnen an vielen Stellen im Modellierungsprozess half:

There was evidence that GeoGebra's features and capabilities assisted students in modeling the real problems, and in making connections between the real world and the mathematical world. Further, the software assisted students in familiarizing themselves with the problem and in broadening their explorations and visualization skills through the process of constructing visual images to analyse the problem, taking into account their informal and visual conceptions (Mousoulides 2011, S. 117).

Besonders aufgefallen in dieser Studie ist dabei die Selbst-Evaluation der Schülerinnen und Schüler, die durch den Gebrauch der Software und deren Werkzeuge permanent die Validität ihrer Lösungen hinterfragten und die Repräsentativität der Modelle in Frage stellten. Dieser Befund konnte von Hertleif und Greefrath (2016) repliziert werden. Dort zeigte sich, dass die Schülerinnen und Schüler die DGS durchgängig zum Bearbeiten einer Modellierungsaufgabe nutzten, sogar für erste Entwürfe und Skizzen. Bei der konkreten Vorgehensweise ließen sich Unterschie- 
de zwischen den Lernenden erkennen. Während einige Schülerinnen und Schüler gezielt nach der Umsetzung eines sich vorher überlegten Modells in der Software suchten, wählten andere die Strategie, sich von den Angeboten der Software inspirieren zu lassen und fuhren mit dem Cursor auf der Suche nach einem geeigneten mathematischen Modell die Werkzeugleiste ab.

All diese Befunde zeigen, dass die Software bei der Bearbeitung einer Modellierungsaufgabe intensiv genutzt wird und dass sich die Modellierungsprozesse mit DGS durchaus von den Prozessen ohne digitales Werkzeug unterscheiden können. Es stellt sich daher die Frage, ob sich diese Unterschiede auch in der Entwicklung von Modellierungskompetenz bemerkbar machen.

\subsection{Lösungsplan}

\subsubsection{Begriffsklärung}

Ein Ansatz für ein Hilfsmittel zum mathematischen Modellierens ist die Nutzung eines strategischen Lösungsplans (Greefrath 2015; Schukajlow et al. 2015a). Strategische Lösungspläne basieren in der Regel auf Schritten im Modellierungskreislauf oder auf Teilkompetenzen, die beim mathematischen Modellieren eine wichtige Rolle spielen. Sie geben einen Überblick über die für die Bearbeitung einer Modellierungsaufgabe sinnvollen Bearbeitungsschritte mit konkreten Hinweisen zu den einzelnen Schritten. Diese sind in der Regel allgemein-strategische Hinweise (vgl. Taxonomie nach Zech 2002), damit der Lösungsplan in allen Inhaltsfeldern eingesetzt werden kann, in seltenen Fällen auch inhaltlich-orientierte Hinweise, die ein bestimmtes mathematisches Thema berücksichtigen. Ein Beispiel für einen Lösungsplan ist in Abb. 3 zu sehen. Neben einem Lösungsplan als zusätzliche metakognitive

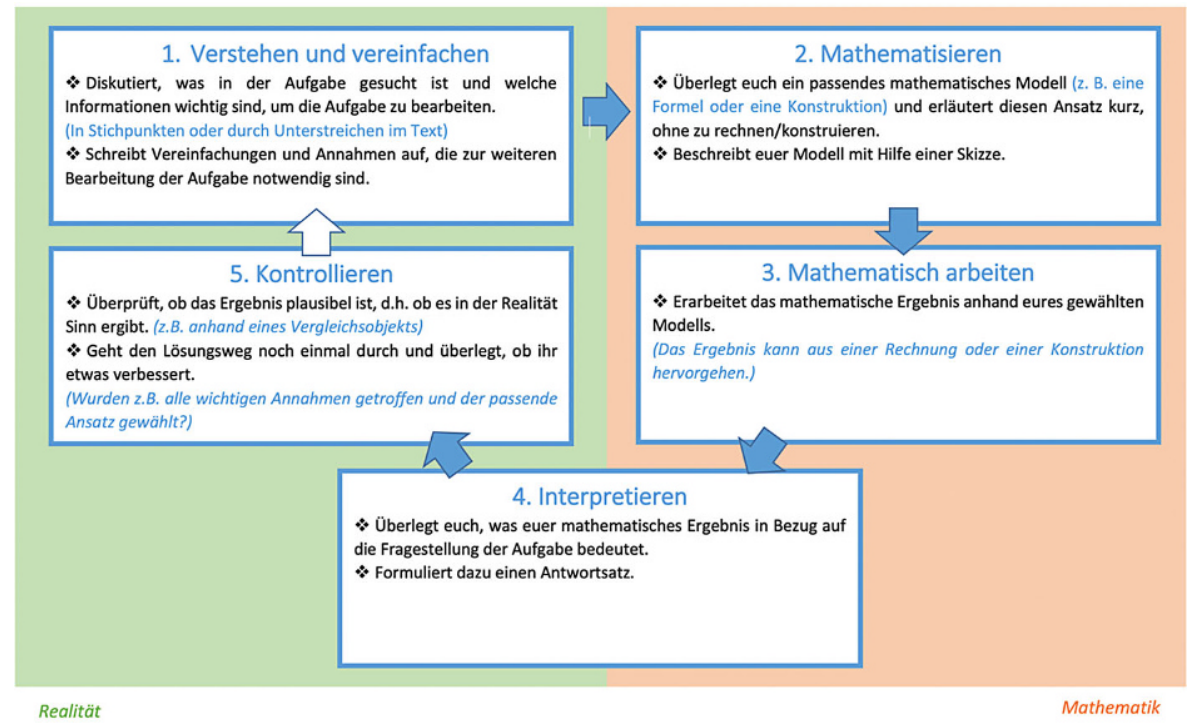

Abb. 3 Lösungsplan im Projekt LIMo (Beckschulte 2019, S. 79) 
Hilfe kann auch ein Arbeitsblatt mit einer entsprechenden Struktur zur Bearbeitung einer Modellierungsaufgabe als Lösungsplan bezeichnet werden. Für die Entwicklung von Metakognition kann der Vergleich und die Diskussion verschiedener Lösungswege und die Vermittlung von Metawissen über Modellierungsprozesse dienen (Maaß 2006). Letzteres ist mit Hilfe eines Lösungsplans gut möglich. Schukajlow und Leiss (2011) nehmen daher in eine Liste für die Bearbeitung von Modellierungsaufgaben nützlicher Strategien auch die Planung des Lösungsprozesses auf. Ein Lösungsplan kann jedoch auch zusätzlichen Lernstoff darstellen und sollte daher nicht zu komplex sein (Meyer und Voigt 2010).

\subsubsection{Lösungsplan-Nutzung beim Modellieren}

In verschiedenen Projekten wurden Lösungspläne für Schülerinnen und Schüler mit unterschiedlicher Länge entwickelt. Einen kurzen Lösungsplan verwenden Zöttl et al. (2010) konkret im Inhaltsbereich Geometrie zum Bearbeiten von Modellierungsaufgaben. Dieser beinhaltet drei Aspekte aus dem Modellierungskreislauf von Blum und Leiß (2005), nämlich Reale Situation, mathematisches Modell und mathematisches Ergebnis.

Der Lösungsplan des DISUM-Projekts (Blum und Schukajlow 2018; Schukajlow et al. 2015a) beschreibt für Lernende adaptiert in verkürzter Form vier Schritte in Anlehnung an Schritte aus dem Modellierungskreislauf von Blum und Leiß (2005) in linearer Form. Dieser Lösungsplan umfasst die vier Schritte: Aufgabe verstehen, Mathematik suchen, Mathematik benutzen und Ergebnis erklären. Jeder Schritt wird den Studierenden anhand von zwei oder drei konkreteren Aufforderungen oder Fragen erläutert. Zum Beispiel soll im zweiten Schritt wichtige Angaben gesucht und der mathematische Zusammenhang zwischen den Angaben beschrieben werden (Blum und Schukajlow 2018, S. 64).

Im Projekt LIMo wurde ein fünfschrittiger Lösungsplan verwendet (Beckschulte 2019). Dieser Lösungsplan umfasst die folgenden Schritte: 1) Verstehen und Vereinfachen, 2) Mathematisieren, 3) Mathematisches Arbeiten, 4) Interpretieren und 5) Kontrollieren (s. Abb. 3) und differenziert so den vierten Schritt des Lösungsplans aus dem DISUM-Projekte in zwei getrennte Schritte auf und betont so das validieren.

\subsubsection{Empirische Befunde zur Lösungsplan-Nutzung beim Modellieren}

In einer qualitativen Studie zur Strategienutzung bei der Bearbeitung von Modellierungsaufgaben konnten unterschiedliche Einschätzungen gefunden werden. Während einige Lernende die Planung des Lösungsprozesses als unabdingbar einschätzen, wird sie von anderen nicht als wichtig eingeschätzt (Stillman und Galbraith 1998). Dieses heterogene Bild steht im Einklang mit Ergebnissen von Schukajlow und Leiss (2011). Dort wird die Planung des Lösungsprozesses von den Lernenden selbst seltener als relevante Lernstrategie im Modellierungsprozess angesehen als andere Lernstrategien. Maßß (2004) hat im Rahmen einer qualitativen Studie in den Klassen 7 und 8 die Modellierungskompetenz von Lernenden untersucht und konnte bei einem großen Teil der Lernenden am Ende der Studie angemessene metakogni- 
tive Modellierungskompetenzen rekonstruieren. Sie beschreibt auch als Ergebnis, dass die Lernenden das Wissen über den Modellierungsprozess und die Darstellung als Kreislauf als Orientierungshilfe empfunden haben.

In einer Studie von Schukajlow et al. (2015a) im Rahmen des DISUM-Projekts konnten in einer 5-stündigen Unterrichtseinheit in Klasse 9 in jeweils drei Klassen mit bzw. ohne Lösungsplan beim mathematischen Modellieren signifikante Unterschiede in den Schülerleistungen nachgewiesen werden, jedoch nur bezogen auf den Inhaltsbereich des Satzes von Pythagoras. Im Inhaltsbereich Lineare Funktionen steigt die globale Modellierungskompetenz sowohl der Gruppe mit als auch ohne Lösungsplan signifikant, jedoch unterscheidet sich die Kompetenzentwicklung in beiden Gruppen nicht. Bezogen auf verschiedene Teilkompetenzen des Modellierens ergibt die Studie von Schukajlow et al. (2015a) für die Teilkompetenz Mathematisieren ähnliche Ergebnisse wie für die globale Modellierungskompetenz. Jedoch hat der Lösungsplan sowohl für den Inhaltsbereich Pythagoras als auch für den Inhaltsbereich Lineare Funktionen einen signifikanten Effekt auf die Teilkompetenz des Interpretierens. Das Lehren mit dem Lösungsplan erwies sich insgesamt als eine effektivere Form des Lehrens und Lernens. Die Lernenden in der Lösungsplangruppe nahmen auch eine stärkere Nutzung von Strategien bzgl. kognitiver Wiederholungs-, Elaborations- und Organisationsstrategien sowie metakognitiver Planungsstrategien wahr (Blum und Schukajlow 2018; Schukajlow et al. 2015a).

Im Rahmen des Projekts KOMMA wurden in 75 achten Klassen an Gymnasien in zwei Bundesländern mit etwa 2000 Schülerinnen und Schülern zusätzlich zum obigen Lösungsplan von Zöttl et al. (2010) fertige Lösungsbeispiele verwendet, die aus einer Problemstellung und der Angabe von Lösungsschritten bestehen. Im Bereich des Mathematischen Begründens und Beweisens konnten bereits positive Effekte beim Einsatz solcher Lösungsbeispiele nachgewiesen werden (Reiss und Renkl 2002). Die Ergebnisse zeigen einen signifikanten Anstieg der Kompetenzen im mathematischen Modellieren (Geometrie) zwischen den Erhebungen vor und nach dem Einsatz der Lernumgebung, der in etwa einem Schuljahr entspricht (Lindmeier et al. 2018; Zöttl et al. 2010).

\section{Fragestellungen}

Bisherige Ergebnisse zum Einsatz unterschiedlicher strategischer Lösungspläne beim Modellieren (Schukajlow et al. 2015a; Zöttl et al. 2010) sowie die Bedeutung metakognitiver Kompetenzen beim Erwerb von Modellierungsprozessen (Stillman 2011) lassen vermuten, dass sich die Modellierungskompetenz durch den Einsatz eines strategischen Lösungsplans fördern lässt.

Auch die Nutzung digitaler Werkzeuge beim Modellieren wird seit langem als förderlich angesehen (Galbraith et al. 2003). Einige Studien zeigen eine vielversprechende Werkzeugnutzung beim Modellieren (Greefrath und Siller 2017; Mousoulides 2011). Auch aktuelle Erkenntnisse über die positiven Auswirkungen von Skizzen und multiplen Lösungen (Rellensmann et al. 2017; Schukajlow et al. 2019) könnten ein Hinweis auf eine mögliche Steigerung der Modellierungsleistung durch DGS sein, die Skizzen und multiple Lösungen unterstützt. 
Sowohl strategische Lösungspläne als auch digitale Werkzeuge wie DGS können Modellierungsprozesse von Lernenden unterstützen. Wenn auch Lösungspläne und DGS nicht grundsätzlich als gleichartige Hilfsmittel strategischer Art gesehen werden können, so kann der Aspekt der metakognitiven Unterstützung durchaus auch in den verschiedenen technischen Werkzeugen einer DGS gesehen werden (vgl. Mousoulides 2011).

In beiden Fällen interessiert insbesondere, welche Teilkompetenzen durch diesen Einsatz jeweils besonders gefördert werden. Hier konzentrieren wir uns auf die Teilkompetenzen Vereinfachen, Mathematisieren, Interpretieren und Validieren. Das mathematische Arbeiten ist weniger typisch für Modellierungsprozesse und die Erhebung von Verstehen und Vermitteln erscheint im Kontext der verwendeten Hilfsmittel weniger relevant. Zunächst ist daher wichtig, ob diese Teilkompetenzen als separate Dimensionen von Modellierungskompetenz erfasst werden können.

Daher stellen wir die folgenden Forschungsfragen:

1. Lassen sich die Teilkompetenzen Vereinfachen, Mathematisieren, Interpretieren und Validieren empirisch als separate Dimensionen eines Kompetenzkonstrukts erfassen?

2. Steigern Schülerinnen und Schüler, die in einer Modellierungseinheit zusätzlich zur Verwendung eines Lösungsplan bzw. einer DGS aufgefordert wurden, stärker ihre Modellierungskompetenz (bzgl. Vereinfachen, Mathematisieren, Interpretieren und Validieren) als Schülerinnen und Schüler in der gleichen Modellierungseinheit ohne Hilfsmittel?

\section{Methodik $^{1}$}

\subsection{Studiendesign}

Um diesen Fragen nachzugehen wurde eine Interventionsstudie mit Prä-, Postund Follow-Up-Test in 44 nordrhein-westfälischen neunten Klassen (Gymnasium, G8 Lehrplan, 45minütige Stunden) durchgeführt. Während der vierstündigen Intervention zum Modellieren nutzten 14 Klassen (283 Personen) durchgängig einen Lösungsplan zur Bearbeitung der Modellierungsaufgaben, 15 Klassen (323 Personen) nutzten durchgängig die DGS GeoGebra und 15 Klassen (315 Personen) arbeiteten ohne ein spezielles Hilfsmittel, sondern nur mit den ,üblichen“ Werkzeugen wie Zirkel und Lineal. Ziel der Studie war es, die Kompetenzentwicklungen in den drei Gruppen (Lösungsplan-, GeoGebra- und Kontrollgruppe) miteinander zu vergleichen. Da die Entwicklung der Teilkompetenzen über die Zeit nicht im Fokus der Studie stand, wurde auf die Integration einer wait-list-Kontrollgruppe, die keinerlei Treatment erfuhr, verzichtet. Die Zuordnung der Klassen auf die drei

\footnotetext{
1 Die hier vorgestellten Erhebungen wurde im Rahmen des Projekts „Lösungsinstrumente beim Modellieren“ (Kurz LIMo) an der WWU Münster durchgeführt. Die verwendeten Daten wurden in zwei eigenständigen Dissertationen (Beckschulte 2019; Hankeln 2019) verwendet. Sie wurden für diesen Artikel zusammengeführt und gemeinsam skaliert und ausgewertet.
} 
Gruppen erfolgte zufällig, soweit äußere Umstände wie etwa das Vorhandensein einer ausreichenden Anzahl funktionierender Computer es erlaubten.

Die Unterrichtsreihe wurde von den regulären Mathematik-Lehrkräften durchgeführt, die vorher speziell für die Studie geschult wurden. Zudem füllten die Lehrkräfte vor Durchführung der Studie einen Fragebogen aus, indem sie eine Selbstauskunft darüber gaben, wie oft sie realitätsbezogene Aufgaben, Aufgaben mit mehreren Lösungswegen und kooperative Arbeitsformen in der betroffenen Klasse einsetzen. Zudem schätzen die Lehrkräfte die Leistungsstärke ihrer Klassen ein. Auch wenn diese Daten nur den subjektiven Eindruck der Lehrkräfte widerspiegeln, wurden sie genutzt, um die Klassen in die Versuchsgruppen einzuteilen, da ein zusätzlicher Test der mathematischen Fähigkeiten zeitlich nicht möglich war. Diese Erhebungen sowie die Gespräche mit den Lehrkräften zeigten, dass die Lernenden zwar mit Realitätsbezügen aus dem Unterricht vertraut waren, ihnen offene Modellierungsaufgaben, die nicht zwingend einen unmittelbaren Bezug zum aktuellen Inhalt herstellen, aber fremd waren. Die Stunden selbst wurden nach einem vorgegebenen Verlaufsplan durchgeführt, dessen Umsetzung stichprobenweise in allen Klassen kontrolliert wurde. Die Lehrkräfte füllten zudem nach jeder Stunde einen Kurzfragebogen zum Verlauf der Stunden aus. Außerdem wurden alle Schülerlösungen eingesammelt und in der DGS-Gruppe auch einige Bildschirme abgefilmt, um analysieren zu können, wie die Lernenden die Software nutzten.

\subsection{Aufgaben der Unterrichtsreihe}

Die Aufgaben der Unterrichtsreihe waren alle sowohl von Hand, als auch mit den beiden Hilfsmitteln (Lösungsplan, DGS) zu bearbeiten. Die Intervention begann mit einer einführenden Stunde, in der die Schülerinnen und Schüler mit Hilfe eines unvollständigen Lösungsbeispiels (Leiss und Tropper 2014; Zöttl 2010) in das Modellieren eingeführt wurden. Inhaltlich wurde in der Stunde die Frage diskutiert, wie viel Rasen zur Erneuerung eines Schlossgartens wohl nötig ist (Hertleif und Adamek 2016). In dieser Aufgabe konnten die Schülerinnen und Schüler der DGS-Gruppe bereits GeoGebra nutzen, die Schülerinnen und Schüler der Lösungsplan-Gruppe arbeiteten hier zunächst mit einem gemäß den Schritten des Lösungsplans vorstrukturierten Arbeitsblatts und wurden anschließend mit dem entsprechenden StrategieInstrument in Papier-Form (s. Abb. 3) vertraut gemacht, das dann ausschließlich weiter genutzt wurde (beispielgebundener Ansatz, vgl. Borromeo Ferri 2018, S. 29). In den folgenden Stunden wurde von den Schülerinnen und Schülern jeweils eine holistische Modellierungsaufgabe mit Hilfe des Lösungsplans in ca. 30-minütiger Partnerarbeit bearbeitet und anschließend im Plenum diskutiert.

Die nachfolgenden Erläuterungen zur Aufgabe „Tower“ (Abb. 4), der dritten Stunde der Intervention, illustriert die Tätigkeiten der Lernenden während der Unterrichtsreihe. Die Aufgabe wurde den Schülerinnen und Schülern im Plenum präsentiert und erste Verständnisfragen geklärt. Die zuvor geschulte Lehrkraft wies bei konkreten Fragen zur Modellierung auf die Eigenverantwortung der Schülerinnen und Schüler hin und informierte sie über ihren Freiraum zu eigenen Entscheidungen. Dann arbeiteten die Lernenden in Paaren an der Aufgabe, dabei stand der Lösungsplangruppe ein Handzettel mit dem Plan zur Verfügung, der GeoGebra- 


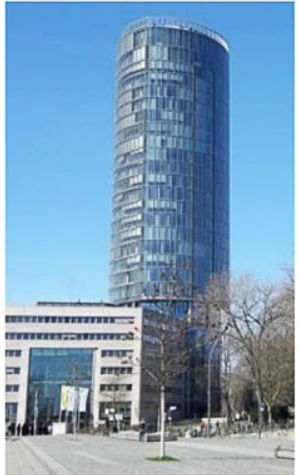

In Köln Deutz steht der sogenannte Köln-Triangle, ein Turm mit ganz besonderer Form. Der 103,20 m große Turm hat 29 Etagen und auf dem Dach eine Aussichtsplattform.

Neben Etagen mit Veranstaltungsräumen und Restaurants gibt es auch einige Etagen, die sich $\mathrm{Ge}$ schäftsleute als Büros mieten können.

Unten seht ihr einen Grundriss einer solchen Büro-Etage, die Aufzüge, das Treppenhaus und die Toiletten abgebildet.

Wie viele Quadratmeter hat eine solche Büro-Etage?

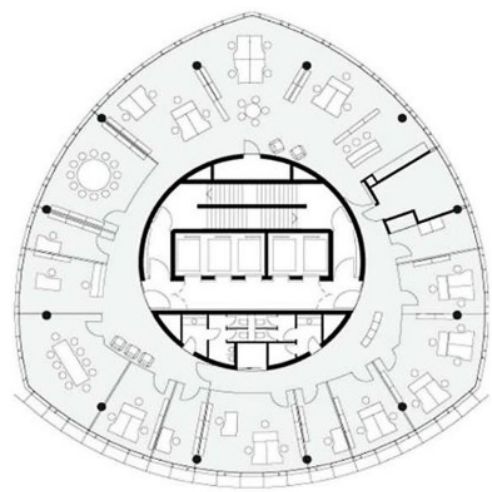

Grundriss: KölnTriangle @ GATERMANN - SCHOSSIG Architekten

Abb. 4 Aufgabe ,Tower“

Gruppe jeweils ein PC pro Paar. Gegen Ende der Stunde präsentierten einige Lernende ihre Vorschläge und die Klasse diskutierte unterschiedliche Ansätze. Dabei achtete die Lehrkraft darauf, dass die Offenheit gewahrt wurde und es nicht Ziel der Diskussion war, nur eine richtige Antwort zu finden.

Im Folgenden wird ein häufig von den Lernenden gewählter Lösungsweg präsentiert, der keinerlei Anspruch erhebt der ideale Weg zu sein. Für andere Vorgehensweisen und knappe Einblicke in Antworten der Lernenden sei auf Hertleif (2018) verwiesen.

Im ersten Schritt müssen die Realsituation und insbesondere der abgebildete Grundriss verstanden werden. So ist zu verstehen, dass die gesamte Etage vermietet wird und diese aus mehreren, unterschiedlich großen Räumen besteht. Außerdem ist eine kreisförmige Mitte zu erkennen, in der Waschräume, Aufzüge und das Treppenhaus zu sehen ist. Die Lernenden müssen demnach die Entscheidung treffen, ob sie annehmen, dass mit der anzumietenden Fläche dieser Bereich gleichermaßen gemeint ist. Gleichzeitig können die Innenwände, Außenbalustrade wie auch andere Angaben aus dem Text, etwa die Höhe und die Etagenanzahl vernachlässigt werden. Um zu einer Größeneinschätzung zu gelangen, kann etwa die Breite eines abgebildeten Tisches oder einer Tür mit $80 \mathrm{~cm}$ als Maßstab verwendet werden.

Die so vereinfachte Realsituation kann dann in ein mathematisches Modell übertragen werden. Beispielsweise kann die Fläche durch ein Reuleaux-Dreieck, also ein gleichseitiges Dreieck mit einbeschriebenen Kreissegmenten auf den Außenseiten angenähert werden. Diese Form entspricht nicht exakt der Form der Etage, kann aber als gute Näherung betrachtet werden. Eine entsprechende Berechnung der Fläche führt zu einem mathematischen Ergebnis, dass dann als ungefähres Ergebnis der Bürofläche genutzt werden kann.

Die so gefundene Größe muss dann auf ihre Plausibilität hin überprüft werden. Dabei ist es besonders hilfreich, die Gesamtfläche durch die Anzahl der Büroräume zu teilen, um eine ungefähre durchschnittliche Bürogröße zu bestimmen und diese mit einer den Schülerinnen und Schülern bekannten Fläche, etwa der Größe ihres Klassenzimmers oder ihres eigenen Zimmers zu vergleichen. 


\subsection{Testinstrument}

Für die vorliegende Studie wurde ein neu entwickelter Test eingesetzt (Hankeln et al. 2019; Hankeln und Beckschulte 2020), der Items zur Messung der Kompetenzen der Schülerinnen und Schüler im Vereinfachen, Mathematisieren, Interpretieren sowie Validieren enthielt. Jeder Test in den insgesamt sechs Testheften (zwei pro Testzeitpunkt) bestand aus vier Blöcken mit je einer Aufgabe für jede der vier Teilkompetenzen, also insgesamt aus 16 Aufgaben. Die Items waren sowohl im Multiple-Choice-, als auch im offenen Antwortformat gestellt. Für den Test wurde ein Multi-Matrix-Design ausgewählt, bei dem nicht jedes Item jeder Schülerin oder jedem Schüler zu jedem Messzeitpunkt alle Items vorgelegt wurden. Da es durch verankerte Testhefte und die Nutzung der Item Response Theorie dennoch möglich war, alle Items auf einer gemeinsamen Skala abzubilden, können durch dieses TestDesign Aussagen über die Entwicklung der Teilkompetenzen gemacht werden, ohne dazu parallele Testhefte konstruieren zu müssen. Gerade bei den kontextgebundenen Modellierungsaufgaben wäre die wirkliche Parallelität von Aufgaben häufig schwierig zu gewährleisten gewesen. Der Test war außerdem ein reiner Paper-Pencil-Test, bei dem die einzelnen Aufgaben nicht als zu einem Teilprozess des Modellierens zugehörig gekennzeichnet waren.

Das Item „Unterlage“ (Abb. 5) zeigt exemplarisch für die Teilkompetenz Validieren, wie die Operationalisierung konkret aussah. ${ }^{2}$ In dieser Aufgabe werden die Schülerinnen und Schülern mit der Aussage konfrontiert, die im Foto abgebildete Schreibtischunterlage habe eine Breite von $40 \mathrm{~cm}$. Die Schülerinnen und Schüler werden dazu aufgefordert, die Plausibilität dieses Ergebnisses mithilfe des Fotos zu überprüfen. In einer korrekten Antwort, die mit zwei Punkten bewertet wurde, wird dabei die Breite von $40 \mathrm{~cm}$ als nicht plausible Lösung erkannt und dies damit begründet, dass beispielsweise das auf der Unterlage zu sehende DIN A4-Blatt bereits eine Breite von $21 \mathrm{~cm}$ besitzt und mehr als zwei Mal (hochkant) auf die Unterlage gelegt werden kann. Eine richtige Antwort erfordert es somit, selbstständig eine geeignete Vergleichsgröße zu finden, wobei dort neben dem DIN A4-Blatt auch ein Vergleich

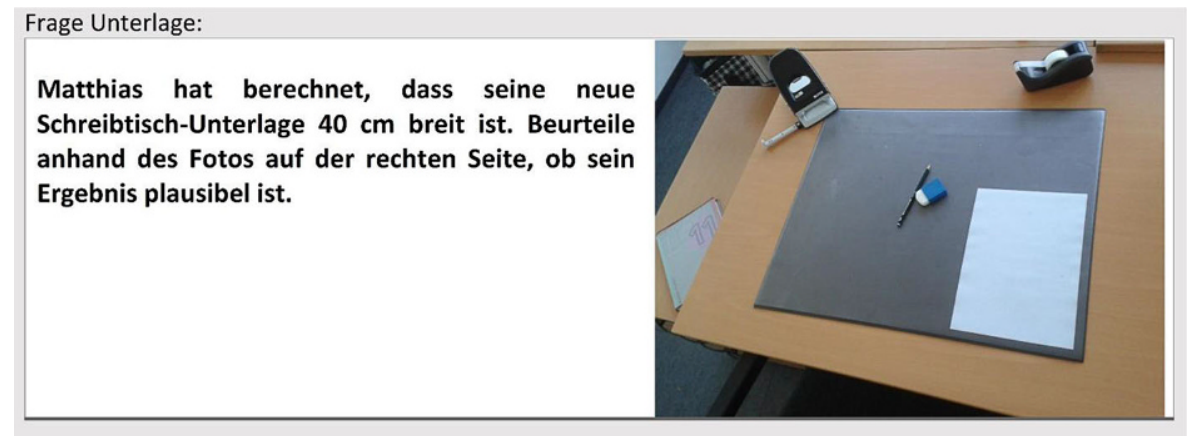

Abb. 5 Item „Unterlage“

2 Die übrigen Testitems sind in Hankeln (2019) sowie Beckschulte (2019) einzusehen. Eine genaue Erläuterung der Testentwicklung ist in Hankeln und Beckschulte (2020) zu finden. 
mit den abgebildeten Stiften möglich wäre, und zudem auch diese Vergleichsgröße in Beziehung zu der Unterlage zu setzen. Damit fragt das Testitem Kompetenzen ab, wie sie auch zur Validierung des Ergebnisses bei der Aufgabe „Tower“ in der Unterrichtsreihe geübt wurden.

Die Testhefte wurden von zwei unabhängigen Ratern unabhängig voneinander mit Hilfe eines in den Pilotierungen entwickelten Kodiermanuals kodiert. Dabei wurden bei einigen Aufgaben auch Teilpunkte für ansatzweise korrekte Lösungen vergeben (Partial-Credit). Die Interrater-Reliabilität (Cohen's Kappa) lag mit 0,81 $\leq \kappa \leq 0,95$ in einem sehr guten Bereich.

\subsection{Auswertungsmethode}

Die so erhobenen Daten wurden gemäß dem einparametrischen Rasch-Modell skaliert. Dabei wurden zunächst zur Beantwortung der ersten Forschungsfrage im Ansatz der ,virtuellen Personen“ (Roeling 2015; Wright 2003) mit den Daten aus allen drei Messzeitpunkten verschiedene Kompetenzmodellierungen berechnet und das am besten passende Modell ausgewählt. Im Ansatz der ,virtuellen Personen“ werden die Daten aus allen Messzeitpunkten untereinander notiert, so dass die gleiche Person in bis zu drei Zeilen der Datenmatrix auftauchen kann. Somit wird in diesem Vorgehen die dem Rasch-Modell zu Grunde liegende Annahme der Unabhängigkeit der Beobachtungen in Bezug auf die Versuchspersonen verletzt. Daher wird dieses Vorgehen nur zur Identifikation des geeigneten Schätzmodells und anschließend zur Bestimmung der Itemparameter des Rasch-Modells genutzt. Zur Bestimmung der Personenparameter wurde die Datenmatrix wieder umstrukturiert und die Daten der drei Messzeitpunkte nebeneinander notiert. Somit wurden in dieser Darstellung statt „,virtueller Personen“, ,,virtuelle Items“ genutzt, da das gleiche Item zu unterschiedlichen Messzeitpunkten als unterschiedliche Beobachtungen gewertet wurde. Für die Bestimmung der Personenparameter wurden daher die in der ersten Skalierung exportierten Itemparameter genutzt (vgl. Roeling 2015). Auf diese Art wurde für jede Person pro gemessene Dimension und Messzeitpunkt ein Fähigkeitsschätzer ermittelt, mit dem anschließend eine zweifaktorielle Varianzanalyse mit Messwiederholung zur Beantwortung der zweiten Forschungsfrage berechnet wurden.

Zur Beantwortung der ersten Forschungsfrage zur separaten Erfassung der Teilkompetenzen wurden unterschiedliche Schätzmodelle im Hinblick auf ihre Passung zu den erhobenen Daten miteinander verglichen (Abb. 6): Zum einen wurde ein eindimensionales Modell bestimmt, bei dem die vier Itemtypen zum Vereinfachen,

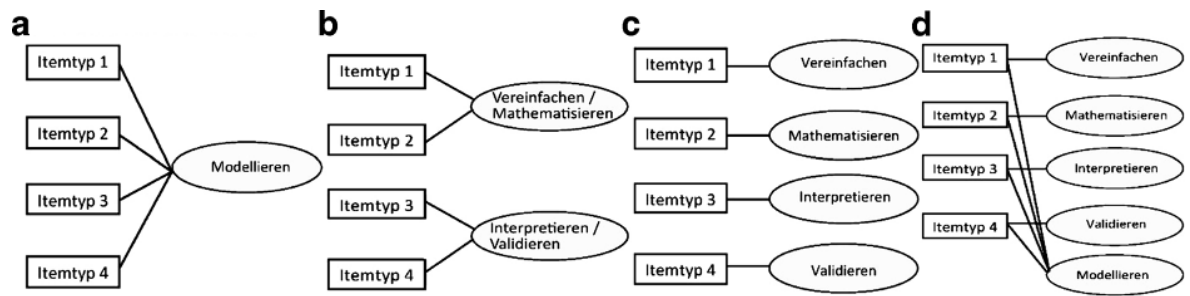

Abb. 6 Verwendete Schätzmodelle, a eindimensionales Modell, b zweidimensionales Modell, c vierdimensionales Modell, d fünfdimensionales Modell 
Mathematisieren, Interpretieren und Validieren alle in etwa das gleiche Konstrukt messen und dementsprechend nur eine Skala bilden. Dem gegenübergestellt wurden zwei weitere between-item-Modelle, bei denen die Itemtypen entweder, wie in den Arbeiten von Zöttl (Zöttl 2010; Zöttl et al. 2011) und Brand (2014) jeweils zwei Teilkompetenzen zu einer Dimension zusammengefasst wurden, oder jede Teilkompetenz eine separate Dimension misst. Außerdem wurde ein within-item-Modell berechnet, bei dem sowohl die Teilkompetenzen als auch eine Gesamtdimension betrachtet werden.

\section{Ergebnisse}

\subsection{Ergebnisse in Bezug auf die Modellierung des Kompetenzkonstrukts}

Die im Ansatz der virtuellen Personen skalierten Modelle wurden im Hinblick auf ihre Passung zu den empirischen Daten miteinander verglichen. Wie Tab. 1 zeigt, fallen für das vierdimensionale Modell sowohl $\mathrm{AIC}^{3}$ als auch $\mathrm{BIC}$ niedriger aus als für alle anderen Modelle. Dies ist insbesondere deshalb von Interesse, da AIC tendenziell zu große (d.h. zu wenig restriktive) Modelle bevorzugt, während BIC zu kleine Modelle präferiert (Kuha 2004). Geben beide Kriterien für dasselbe Modell einen kleineren Wert aus, so kann dieses Modell angenommen werden, da es laut Kuha (2004) ,unlikely to be far from the best of the candidate models“ (S. 223) ist. Somit bestätigt sich, dass das vierdimensionale Modell, bei dem jede der Teilkompetenzen eine eigenständige Dimension bildet, relativ am besten auf die erhobenen Daten passt.

Auf Ebene der einzelnen Items liegen die Weighted Mean Squares im vierdimensionalen Modell zwischen 0,93 und 1,12, also klar innerhalb der von Bond und Fox (2007) formulierten Grenzen von 0,8 bis 1,2. Daher wurden keine Items ausgeschlossen. Die Item-Total-Korrelationen, die im dichotomen Fall der Trennschärfe entsprechen, liegen zwischen 0,21 und 0,52, im Durchschnitt bei 0,39 und sind somit als akzeptabel zu werten.

Tab. 1 Passung der verschiedenen Kompetenzmodelle

\begin{tabular}{lllll}
\hline Modell & Eindimensional & Zweidimensional & Vierdimensional & Fünfdimensional \\
\hline Stichprobengröße & 3028 & 3028 & 3028 & 3028 \\
Final deviance & 77739,70934 & 77739,81459 & 77436,19113 & 77436,63903 \\
Anzahl Parameter & 52 & 54 & 61 & 66 \\
AIC & 77843,70934 & 77847,81459 & 77558,19113 & 77568,63903 \\
BIC & 78208,52354 & 78226,66010 & 77986,14624 & 78031,67243 \\
\hline
\end{tabular}

\footnotetext{
3 Üblicherweise werden verschiedene Kriterien zur Überprüfung der Modellgüte betrachtet, welche die Likelihood-Funktion, die Parameterzahl $\mathrm{n}$ und die Stichprobengröße $\mathrm{N}$ in unterschiedlicher Gewichtung berücksichtigen: Das Akaike Information Criterion $(\mathrm{AIC}=-2 \log (\mathrm{L})+2 \mathrm{n})$ und das Bayes Information Criterion $(\mathrm{BIC}=-2 \log (\mathrm{L})+\log (\mathrm{N}) \mathrm{n})$. Beide Prüfgrößen haben gemein, dass ein kleiner Wert eine bessere Modellpassung angibt als ein großer Wert, allerdings ohne dass es eine Mindestgröße für den Unterschied der jeweiligen Werte gibt (vgl. Rost 2004, S. 341).
} 


\subsection{Ergebnisse in Bezug auf die Teilkompetenzentwicklung}

Nachdem das vierdimensionale Modelle als das für die Bestimmung der Personenparameter am geeignetste identifiziert wurde, wurde pro Person für jede Teilkompetenz und jeden Messzeitpunkt ein Fähigkeitsparameter bestimmt. In Tab. 2 ist zu sehen, dass die Personenfähigkeiten mit einer EAP/PV-Reliabilität zwischen 0,60 und 0,72 bestimmt werden konnte. Die EAP/PV-Reliabilität vergleicht die erwartete mit der tatsächlichen Varianz und ist daher mit Cronbachs Alpha vergleichbar. Die erreichten Werte sind also als ausreichend für Gruppenvergleiche einzuschätzen. Tab. 3 zeigt die jeweiligen Mittelwerte und Standardabweichungen in den drei Gruppen.

Die zweifaktorielle Varianzanalyse (Tab. 4) zeigt, dass der Innersubjektfaktor, das heißt die Entwicklung über die Zeit, in allen Teilkompetenzen signifikant wird. Zudem ist die Interaktion zwischen den Messzeitpunkten und der Versuchsgruppe beim Mathematisieren und Interpretieren signifikant. Beim Validieren ist der Zwischensubjektfaktor Versuchsgruppe signifikant.

Betrachtet man die Entwicklung über die Zeit noch einmal differenziert für die verschiedenen Gruppen, so zeigt sich, dass vom ersten zum zweiten Messzeit-

Tab. 2 EAP/PV-Reliabilitäten der Personenparameter

\begin{tabular}{llll}
\hline 3 & Messzeitpunkt 1 & Messzeitpunkt 2 & Messzeitpunkt 3 \\
\hline Vereinfachen & 0,719 & 0,619 & 0,648 \\
Mathematisieren & 0,699 & 0,626 & 0,661 \\
Interpretieren & 0,656 & 0,646 & 0,709 \\
Validieren & 0,610 & 0,679 & 0,602 \\
\hline
\end{tabular}

Tab. 3 Mittelwerte und Standardabweichung (SD) der Fähigkeitsparameter zu den verschiedenen Messzeitpunkten

\begin{tabular}{|c|c|c|c|c|c|c|c|}
\hline & & \multicolumn{2}{|c|}{ Messzeitpunkt 1} & \multicolumn{2}{|c|}{ Messzeitpunkt 2} & \multicolumn{2}{|c|}{ Messzeitpunkt 3} \\
\hline & & Mittelwert & SD & Mittelwert & $\mathrm{SD}$ & Mittelwert & SD \\
\hline \multicolumn{8}{|c|}{ Vereinfachen } \\
\hline \multirow{3}{*}{$\begin{array}{l}\text { Versuchs- } \\
\text { grup- } \\
\text { pe }\end{array}$} & Kontroll & $-0,08$ & 0,80 & 0,00 & 0,81 & $-0,03$ & 0,88 \\
\hline & GeoGebra & $-0,01$ & 0,78 & 0,03 & 0,86 & 0,07 & 0,82 \\
\hline & Lösungsplan & $-0,10$ & 0,79 & 0,06 & 0,87 & 0,02 & 0,85 \\
\hline \multicolumn{8}{|c|}{ Mathematisieren } \\
\hline \multirow{3}{*}{$\begin{array}{l}\text { Versuchs- } \\
\text { gruppe }\end{array}$} & Kontroll & $-0,15$ & 1,04 & 0,08 & 1,04 & $-0,17$ & 1,11 \\
\hline & GeoGebra & 0,04 & 1,08 & 0,01 & 1,05 & 0,01 & 1,23 \\
\hline & Lösungsplan & $-0,04$ & 1,07 & $-0,03$ & 1,08 & $-0,15$ & 1,09 \\
\hline \multicolumn{8}{|c|}{ Interpretieren } \\
\hline \multirow{3}{*}{$\begin{array}{l}\text { Versuchs- } \\
\text { gruppe }\end{array}$} & Kontroll & $-0,34$ & 1,21 & 0,03 & 1,29 & $-0,16$ & 1,42 \\
\hline & GeoGebra & $-0,18$ & 1,24 & $-0,08$ & 1,23 & 0,13 & 1,45 \\
\hline & Lösungsplan & $-0,30$ & 1,25 & 0,08 & 1,22 & 0,09 & 1,43 \\
\hline \multicolumn{8}{|c|}{ Validieren } \\
\hline \multirow{3}{*}{$\begin{array}{l}\text { Versuchs- } \\
\text { gruppe }\end{array}$} & Kontroll & $-0,20$ & 0,73 & 0,04 & 0,84 & $-0,03$ & 0,90 \\
\hline & GeoGebra & $-0,06$ & 0,74 & 0,15 & 0,88 & 0,11 & 0,81 \\
\hline & Lösungsplan & $-0,10$ & 0,72 & 0,08 & 0,78 & 0,01 & 0,88 \\
\hline
\end{tabular}


Tab. 4 Ergebnisse der zweifaktoriellen Varianzanalyse mit Messwiederholung (MZP= Messzeitpunkt, Vgrp = Versuchsgruppe)

\begin{tabular}{llllllllllllll}
\hline & \multicolumn{3}{c}{ Vereinfachen } & \multicolumn{4}{c}{ Mathematisieren } & \multicolumn{3}{c}{ Interpretieren } & \multicolumn{4}{c}{ Validieren } \\
& $\mathrm{F}$ & $p$ & $\eta^{2}$ & $\mathrm{~F}$ & $p$ & $\eta^{2}$ & $\mathrm{~F}$ & $p$ & $\eta^{2}$ & $\mathrm{~F}$ & $p$ & $\eta^{2}$ \\
\hline MZP & 7,195 & 0,001 & 0,008 & 5,397 & 0,005 & 0,006 & 25,588 & 0,000 & 0,027 & 26,408 & 0,000 & 0,028 \\
MZP* & 0,663 & 0,617 & 0,001 & $\mathbf{2 , 6 9 6}$ & 0,020 & 0,006 & 4,170 & 0,002 & 0,009 & 0,473 & 0,756 & 0,001 \\
Vgrp & & & & & & & & & & & & \\
Vgrp & 1,044 & 0,353 & 0,002 & 1,360 & 0,257 & 0,003 & 1,174 & 0,310 & 0,003 & 3,592 & 0,028 & 0,008 \\
\hline
\end{tabular}

Tab. 5 Veränderung der Teilkompetenzen in den Versuchsgruppen

\begin{tabular}{llllllllllllllll}
\hline Messzeitpunkt 1 zu 2 & \multicolumn{1}{c}{ Kontrollgruppe } & \multicolumn{1}{c}{ GeoGebra-Gruppe } & \multicolumn{4}{c}{ Lösungsplangruppe } \\
& Diff & $T$ & $p$ & $d$ & Diff & $T$ & $p$ & $d$ & Diff & $T$ & $p$ & $d$ \\
Ver & $+0,08$ & 1,57 & 0,118 & 0,14 & $+0,04$ & 0,75 & 0,453 & 0,07 & $+0,16$ & 2,84 & 0,005 & 0,27 \\
Math & $+0,23$ & 3,63 & 0,000 & 0,31 & $-0,03$ & $-0,43$ & 0,669 & 0,04 & $+0,01$ & 0,15 & 0,883 & 0,01 \\
Int & $+0,36$ & 4,82 & 0,000 & 0,42 & $+0,11$ & 1,34 & 0,166 & 0,04 & $+0,38$ & 4,27 & 0,000 & 0,31 \\
Val & $+0,24$ & 5,01 & 0,000 & 0,34 & $+0,21$ & 4,17 & 0,000 & 0,37 & $+0,18$ & 3,53 & 0,000 & 0,44
\end{tabular}

\section{Messzeitpunkt 2 zu 3}

\begin{tabular}{|c|c|c|c|c|c|c|c|c|c|c|c|c|}
\hline & \multicolumn{4}{|c|}{ Kontrollgruppe } & \multicolumn{4}{|c|}{ GeoGebra-Gruppe } & \multicolumn{4}{|c|}{ Lösungsplangruppe } \\
\hline & Diff & $T$ & $p$ & $d$ & Diff & $T$ & $p$ & $d$ & Diff & $T$ & $p$ & $d$ \\
\hline Ver & $-0,02$ & $-0,45$ & 0,651 & 0,05 & $+0,04$ & 0,79 & 0,433 & 0,07 & $-0,04$ & 0,79 & 0,433 & 0,07 \\
\hline Math & $-0,28$ & $-4,00$ & 0,000 & 0,33 & $-0,00$ & $-0,06$ & 0,956 & 0,00 & $-0,12$ & $-1,61$ & 0,108 & 0,16 \\
\hline Int & $-0,19$ & $-2,37$ & 0,018 & 0,20 & $+0,24$ & 2,97 & 0,003 & 0,22 & $+0,01$ & 0,13 & 0,897 & 0,01 \\
\hline Val & $-0,11$ & $-1,96$ & 0,051 & 0,11 & $-0,05$ & $-0,99$ & 0,324 & 0,07 & $-0,07$ & $-1,26$ & 0,208 & 0,12 \\
\hline \multicolumn{13}{|c|}{ Messzeitpunkt 1 zu 3} \\
\hline & \multicolumn{4}{|c|}{ Kontrollgruppe } & \multicolumn{4}{|c|}{ GeoGebra-Gruppe } & \multicolumn{4}{|c|}{ Lösungsplangruppe } \\
\hline & Diff & $T$ & $p$ & $d$ & Diff & $T$ & $p$ & $d$ & Diff & $T$ & $p$ & $d$ \\
\hline Ver & $+0,08$ & 1,50 & 0,134 & 0,08 & $+0,09$ & 1,82 & 0,070 & 0,14 & $+0,12$ & $-2,331$ & 0,020 & 0,21 \\
\hline Math & $-0,05$ & $-0,71$ & 0,477 & 0,03 & $-0,03$ & $-0,43$ & 0,668 & 0,04 & $-0,11$ & 1,604 & 0,110 & 0,14 \\
\hline Int & $+0,20$ & 2,41 & 0,016 & 0,19 & $+0,33$ & 3,92 & 0,000 & 0,32 & $+0,39$ & $-4,607$ & 0,000 & 0,41 \\
\hline Val & $+0,17$ & 3,18 & 0,002 & 0,29 & $+0,14$ & 3,00 & 0,003 & 0,31 & $+0,12$ & $-1,986$ & 0,048 & 0,19 \\
\hline
\end{tabular}

punkt alle drei Versuchsgruppen ihre Fähigkeiten im Validieren signifikant steigern (Tab. 5). Die Kontrollgruppe steigert zudem noch ihre Fähigkeiten im Mathematisieren und Interpretieren signifikant, in der Lösungsplangruppe werden die Fähigkeiten im Vereinfachen und Interpretieren signifikant gesteigert. Einzig die GeoGebraGruppe weist keine weiteren Veränderungen auf.

Betrachtet man die Entwicklung vom zweiten zum dritten Messzeitpunkt, so findet einzig in der Lösungsplangruppe keinerlei signifikante Veränderung statt. In der Kontrollgruppe hingegen sinken die Fähigkeiten im Mathematisieren und Interpretieren wieder signifikant. In der GeoGebra-Gruppe hingegen ist eine signifikante Steigerung der Fähigkeiten im Interpretieren zu erkennen.

In Bezug auf die langfristige Entwicklung vom ersten zum dritten Messzeitpunkt ist festzustellen, dass alle Gruppen eine signifikante Verbesserung im Bereich des In- 
terpretierens und Validierens erfahren haben. Einzig die Lösungsplangruppe konnte zudem ihre Fähigkeiten im Vereinfachen nachhaltig steigern.

\section{Interpretation und Diskussion}

Das erste Erkenntnisinteresse der vorgestellten Studie liegt in der Fragestellung, wie sich die Kompetenzstruktur des mathematischen Modellierens empirisch beschreiben lässt. Diese Frage greift damit die bei Blum (2002) formulierte Frage auf, ob es möglich ist, spezielle Teilfähigkeiten oder Teilkompetenzen einer (umfassenderen) Modellierungskompetenz zu identifizieren. Wie eingangs beschrieben beschränkt sich die LIMo-Studie dabei allerdings auf vier unterschiedliche Komponenten von Modellierungskompetenz.

Zunächst ist festzuhalten, dass mit Hilfe des neu entwickelten Testinstruments die betrachteten vier Teilkompetenzen Vereinfachen, Mathematisieren, Interpretieren und Validieren mit ausreichender Reliabilität erfasst werden konnten. Es konnte weiterhin durch die Skalierung mit dem Ansatz der virtuellen Personen gezeigt werden, dass sich die erhobenen Daten am besten durch ein vierdimensionales between-itemModell beschreiben lassen, bei dem die verschiedenen Teilkompetenzen als separate Dimensionen eines latenten Konstrukts erfasst werden. Daraus lässt sich folgern, dass die betrachteten Teilkompetenzen auch empirisch messbare unterschiedliche Leistungsdispositionen darstellen. Die in der Literatur genannten Teilkompetenzen Vereinfachen, Mathematisieren, Interpretieren und Validieren können somit zwar als unterschiedliche Komponenten einer globaleren Modellierungskompetenz verstanden werden, jedoch ist es möglich, dass es weitere Komponenten einer solchen Modellierungskompetenz gibt. Dieses Resultat lässt sich wie folgt in die bestehende Forschung einordnen: Die vorliegende Studie bestätigt die Erkenntnisse von Brand (2014) und Zöttl (2010) bezüglich des Komponenten-Modells insofern, dass ein atomistisch aufgebauter Modellierungstest dazu in der Lage ist, statt einer holistischen Kompetenz, mehrere Teilkompetenzen zu messen. Dabei ist allerdings zu bemerken, dass in die Studien von Brand (2014) und Zöttl (2010) auch Aufgaben zur Messung der (holistischen) Modellierungskompetenz eingesetzt wurden und eine weitere Dimension darstellten. Neu an der vorliegenden Studie ist es, dass auch die Teilkompetenzen des Vereinfachens und Mathematisierens sowie des Interpretierens und des Validierens bei der Messung voneinander separiert wurden. Dieser Ansatz ist jedoch nicht unstrittig zu sehen. So zeigen die Befunde von Borromeo Ferri (2006), dass in tatsächlichen Modellierungsprozessen die unterschiedlichen Teilprozesse oder Modelle aus qualitativer Perspektive gar nicht eindeutig voneinander zu trennen sind. Die Schwierigkeiten der Trennung der Teilphasen bei Borromeo Ferri (2006) nehmen Djepaxhija et al. (2016) zum Anlass, gar keine Trennung von Teilprozessen vorzunehmen, sondern alle Prozesse vom Verstehen über das Vereinfachen und Strukturieren bis zum Aufstellen eines mathematischen Modells unter dem Begriff Mathematisieren zusammenfassen. Eine solche weitgreifende Kompetenzauffassung kann jedoch die Beurteilung der Wirkung von Bildungsmaßnahmen deutlich erschweren, da die Ursachen für beobachtete Unterschiede vielfältig sein können. Bei der Evaluation von Bildungsmaßnahmen, wie etwa bei Interventions- 
studien ist daher eine genaue Diagnose wünschenswert, an welchen Stellen Effekte sichtbar werden (vgl. Hartig 2006).

In Bezug auf die zweite Forschungsfrage lässt sich festhalten, dass eine Veränderung der Kompetenzwerte über die Zeit hinweg stattgefunden hat. Allerdings fielen nur in den Teilkompetenzen Interpretieren und Mathematisieren Unterschiede in der Entwicklung der verschiedenen Versuchsgruppen auf, das allerdings auch mit sehr geringer Effektstärke. Im Bereich Vereinfachen war insgesamt eine eher geringe Veränderung der Kompetenzwerte zu beobachten. Einzig der Lösungsplangruppe gelang es deutlich, ihre Kompetenzen zu steigern. Diese Steigerung war sogar nachhaltig, denn zwischen dem zweiten und dritten Messzeitpunkt fand keine signifikante Abnahme mehr statt, so dass sich die Fähigkeiten der Lösungsplangruppe im Bereich Vereinfachen vom ersten zum dritten Messzeitpunkt signifikant verbessert haben, allerdings nur mit kleiner Effektstärke (vgl. Tab. 5).

Eine mögliche Erklärung dafür, dass nur die Lösungsplangruppe ihre Kompetenzen in diesem Bereich besonders steigern konnte, könnte die verstärkte Fokussierung der Lernenden auf diesen Schritt durch die Verwendung eines Lösungsplans sein. Es ist bereits bekannt, dass Lernende häufig vorschnell anfangen ein mathematisches Modell zu bilden ohne sich ausreichend Gedanken über die vorliegende Situation zu machen (Leiss et al. 2010). Möglicherweise führte die Verwendung des Lösungsplans zu einem reflektierteren Vorgehen und damit zu einem nachhaltigeren Lernen.

Im Bereich Mathematisieren steigert im Verlauf der Unterrichtseinheit nur die Kontrollgruppe ihre Kompetenzen signifikant. Allerdings ist diese Verbesserung nicht nachhaltig, das heißt zum dritten Messzeitpunkt nicht mehr erkennbar. Bei der Einschätzung dieses Unterschieds ist aber zu beachten, dass die Kontrollgruppe im Prätest einen deutlich geringeren Kompetenzwert aufweist als die übrigen Gruppen, was einen Einfluss auf die Veränderung gehabt haben kann. ${ }^{4}$ Dass die GeoGebra-Gruppe keinen signifikanten Zuwachs aufweist, kann außerdem daran liegen, dass die Testung des Mathematisierens auf Papier sich zu stark von dem Erstellen eines Modells in der Software unterschied. So standen den Lernenden der GeoGebra-Gruppe stets verschiedene Ideen in der Werkzeugleiste des Programms zur Verfügung, die bei dem Test auf Papier nicht vorhanden waren.

Im Bereich Interpretieren konnten vom ersten zum zweiten Messzeitpunkt nur die Kontroll- sowie die Lösungsplangruppe ihre Kompetenzen signifikant steigern. Zwar ist auch bei der GeoGebra-Gruppe ein Anstieg zu erkennen, dieser konnte allerdings nicht gegen den Zufall abgesichert werden. Eine mögliche Erklärung dafür ist, dass die GeoGebra-Gruppe während der Intervention nicht zwingend ein rein mathematisches Modell interpretieren, sondern jeweils ein digitales Modell zurück in die Realität übertragen musste. Daher ist es umso erstaunlicher, dass gerade diese Gruppe einen signifikanten Zuwachs vom zweiten zum dritten Messzeitpunkt aufweist. Allerdings konnte für diesen Zeitraum nicht kontrolliert werden, was jeweils im Unterricht thematisiert wurde, sodass diese Entwicklung nicht überinterpretiert werden sollte. Interessant ist allerdings, dass der signifikante Zuwachs einzig bei der Lösungsplangruppe nachhaltig, das heißt noch zum dritten Messzeitpunkt nachweisbar

\footnotetext{
${ }^{4}$ Für eine Auswertung mittels Regressionsanalysen, bei denen für den ersten Messzeitpunkt kontrolliert wurde, sei auf Hankeln (2019) verwiesen.
} 
war. Es ist möglich, dass die Bewusstmachung der einzelnen Modellierungsschritte in diesem Fall zu einem besser verankerten Kompetenzaufbau geführt hat.

In Bezug auf die Teilkompetenz Validieren konnten alle drei Versuchsgruppen ihre Fähigkeiten signifikant steigern. Dies liegt vermutlich auch daran, dass es sich bei dieser Teilkompetenz um eine häufig eher etwas vernachlässigte Fähigkeit im Mathematikunterricht handelt. Vom ersten zum zweiten Messzeitpunkt konnte die Lösungsplangruppe ihre Fähigkeiten mit der größten Effektstärke steigern, gefolgt von der GeoGebra-Gruppe. In Bezug auf die Nachhaltigkeit dieser Steigerung weist die GeoGebra-Gruppe den größten Effekt auf. Diese Steigerungen lassen sich möglicherweise unterschiedlich erklären. Während der Lösungsplan den Lernenden den Schritt des Validierens als notwendigen Schritt deutlich macht, regt die Software durch das leichte Rückgängig-Machen und die Vielzahl an Modellen besonders das Ausprobieren und Vergleichen verschiedener Modelle an. Diese Überlegungen weisen darauf hin, dass eine mögliche Kombination von Lösungsplan und DGS ebenfalls erfolgsversprechend sein könnte, ähnlich wie die von Haug (2008) eingesetzten Reflexionsfragen bei der Arbeit mit einer DGS.

Die oben präsentierten Befunde sind jedoch immer vor dem methodischen Hintergrund der Studie zu interpretieren. Hier spielt auch die Frage eine Rolle, in wie weit die beiden Hilfen miteinander vergleichbar sind. Wenn auch DGS etwa durch eine umfangreiche Werkzeugleiste durchaus metakognitive Unterstützung anregen kann (Hertleif und Greefrath 2016; Mousoulides 2011), so gibt es hier weitere Aspekte als Werkzeug, die beim strategischen Lösungsplan nicht gegeben sind. So kann das digitale Werkzeug etwa stärker beim Berechnen unterstützen, auch wenn das bei den gewählten Aufgaben weniger eine Rolle spielt. Da die Betrachtung der schriftlichen Schülerlösungen sowie die Analyse der punktuell durchgeführten BildschirmVideographien zeigten, dass alle Lernenden dieser Gruppe die DGS zur Lösungsfindung nutzten und durchaus auch unterschiedliche Modelle entwickelten, können die beobachteten Effekte durchaus auf das eingesetzte Werkzeug zurückgeführt werden. Das Arbeitsblatt nutzten die Schülerinnen und Schüler der DGS-Gruppe nur für Notizen oder zur Dokumentation ihrer in der DGS gefundenen Lösung.

Bei der Durchführung im Feld mussten einige Kompromisse eingegangen werden. Zum einen konnten die Lernereigenschaften nicht vollständig erfasst werden. Zwar wurden einige Variablen wie Vorwissen, Geschlecht, Sicherheit und Einstellungen mit und zur Software erfasst und deren Einfluss kontrolliert. Sicherlich könnten aber auch Lernpräferenzen oder Denkstile (vgl. Borromeo Ferri 2010) Einfluss auf den Erfolg der eingesetzten Unterrichtsreihe gehabt haben.

Ein weiterer nicht zu vernachlässigender Aspekt ist die Rolle der Lehrkraft während der Unterrichtseinheit. Wie die Hattie-Studie (Hattie 2010) sowie die CoActivStudie (z.B. Baumert et al. 2010) zeigen, hat die Lehrkraft durch ihr Wissen, ihr Verhalten und ihre Kompetenzen entscheidenden Einfluss auf die Lernleistung der Schülerinnen und Schüler. Da dieser Einfluss aber nicht Untersuchungsgegenstand dieser Studie war, sollte er möglichst konstant gehalten werden. Idealerweise hätte also eine unabhängige Lehrkraft in allen Klassen die Projektstunden unterrichtet. Dies war bei den 44 Klassen aus ganz NRW, die insgesamt am LIMo-Projekt teilnahmen, aber nicht realisierbar. Der Einsatz einer fremden Lehrkraft hätte zudem den Nachteil gehabt, dass die Schülerinnen und Schüler sich an einen neuen Unterrichts- 
stil und eine neue Person hätten gewöhnen müssen, was ebenfalls einen Einfluss auf ihr Lernverhalten hätte haben können. Somit wäre diese Designentscheidung der externen Validität und damit der Übertragbarkeit auf gewöhnliche Unterrichtssituationen abträglich gewesen. Daher wurde stattdessen versucht, darauf zu achten, durch die Verteilung der Lehrkräfte keine systematische Verzerrung der Versuchsgruppen zu bewirken, sondern die Klassen samt der Lehrperson möglichst zufällig auf die Gruppen zu verteilen. Es wurde weiterhin versucht, der Beeinflussung dadurch entgegenzuwirken, dass alle Lehrkräfte möglichst umfassend geschult und auf die Projektstunden vorbereitet wurden. Durch die Besprechung des umfangreichen Materials, das auf den Erfahrungen aus den Pilotierungen der Studie aufbaute, wurden den Lehrkräften konkrete Handlungsanweisungen sowie detaillierte Ablaufpläne an die Hand gegeben. Die Umsetzung wurde stichprobenartig kontrolliert.

Bei der Auswahl der Stichprobe handelt es sich also nicht um eine Zufallsauswahl, sondern um Beteiligung ganzer Klassen, deren Lehrkräfte für die Teilnahem an der Studie gewonnen werden konnten. Die tatsächliche Nutzung der zur Verfügung gestellten Hilfsmittel konnte nicht erfasst werden. Allerdings wurden in allen Gruppen die entstandenen Produkte, in der DGS-Gruppe auch die digitalen Dateien, eingesammelt und auf Auffälligkeiten hin untersucht.

Ein weiterer Kritikpunkt, der der Durchführbarkeit im Schulalltag geschuldet ist, ist die Dauer der Intervention. Es handelt sich um eine relativ kurze Intervention von vier Unterrichtsstunden. Daher waren bereits a priori keine sehr großen Veränderungen $\mathrm{zu}$ erwarten gewesen. Jedoch wurde in diesen Stunden einzig das Modellieren thematisiert, was sonst nur selten in diesem Umfang im Regelunterricht geschieht. So war auch die inhaltliche Breite der verwendeten Modellierungsaufgaben begrenzt und die unterrichtlichen Aktivitäten zwischen Posttest und Follow-up-Test konnten nicht kontrolliert werden.

\section{Fazit}

Es kann festgestellt werden, dass durch die durchgeführte Unterrichtseinheit mit vier komplexen Modellierungsaufgaben die Modellierungskompetenz, insbesondere das Validieren, gesteigert werden konnte. Jedoch war die Steigerung gering und die Unterschiede zwischen den Gruppen waren nur in bestimmten Teilkompetenzen sichtbar. Für die Förderung des Vereinfachens verspricht ein Lösungsplan Erfolge. Insgesamt ist zu bedenken, dass die Nutzung der Hilfsmittel langfristig eingeübt werden muss, um dann später im Sinne eines Scaffolding (Schukajlow et al. 2015a) wieder zurückgeführt werden zu können. Für die Praxis ist es auch ein interessantes Ergebnis, dass der Kompetenzzuwachs beim Modellieren durch die zusätzliche Nutzung digitaler Werkzeuge nicht sinkt und somit digitale Werkzeuge und Modellieren gleichzeitig gelernt werden können.

Für weitere Studien liegt nun ein Testinstrument zur mathematischen Modellierungskompetenz von Schülerinnen und Schülern vor, das mit Hilfe von atomistischen Modellierungsaufgaben die vier zentralen Teilkompetenzen Vereinfachen, Mathematisieren, Interpretieren und Validieren getrennt erfassen kann. Hier könnte nun die Chance genutzt werden, längere Interventionen durchzuführen, um so mög- 
licherweise deutlichere Effekte zu beobachten. Für die Nutzung von DGS könnten die Ergebnisse durch Erhebung von Prozessdaten genauer analysiert werden und mit den Ergebnissen qualitativer Studien gemeinsam diskutiert werden. Ggf. müsste auch über die Kompetenzmessung mit Hilfe der DGS nachgedacht werden. Interessant könnten auch Überlegungen sein, verschieden Hilfsmittel beim Modellieren miteinander zu kombinieren und aufeinander abzustimmen.

Danksagung Wir bedanken uns bei Catharina Beckschulte für die Möglichkeit, die Daten zur Lösungsplangruppe für die Auswertung zu nutzen.

Funding Open Access funding enabled and organized by Projekt DEAL.

Open Access Dieser Artikel wird unter der Creative Commons Namensnennung 4.0 International Lizenz veröffentlicht, welche die Nutzung, Vervielfältigung, Bearbeitung, Verbreitung und Wiedergabe in jeglichem Medium und Format erlaubt, sofern Sie den/die ursprünglichen Autor(en) und die Quelle ordnungsgemäß nennen, einen Link zur Creative Commons Lizenz beifügen und angeben, ob Änderungen vorgenommen wurden.

Die in diesem Artikel enthaltenen Bilder und sonstiges Drittmaterial unterliegen ebenfalls der genannten Creative Commons Lizenz, sofern sich aus der Abbildungslegende nichts anderes ergibt. Sofern das betreffende Material nicht unter der genannten Creative Commons Lizenz steht und die betreffende Handlung nicht nach gesetzlichen Vorschriften erlaubt ist, ist für die oben aufgeführten Weiterverwendungen des Materials die Einwilligung des jeweiligen Rechteinhabers einzuholen.

Weitere Details zur Lizenz entnehmen Sie bitte der Lizenzinformation auf http://creativecommons.org/ licenses/by/4.0/deed.de.

\section{Literatur}

Adan, I. J. B. F., Perrenet, J. C., \& Sterk, H. J. M. (2005). Da kracht van wiskundig modelleren. Eindhoven: Technische Universiteit Eindhoven.

Baumert, J., Kunter, M., Blum, W., Brunner, M., Voss, T., Jordan, A., et al. (2010). Teachers' mathematical knowledge cognitive activation in the classroom, and student progress. American Educational Research Journal. https://doi.org/10.3102/0002831209345157.

Beckschulte, C. (2019). Mathematisches Modellieren mit Lösungsplan: Eine empirische Untersuchung zur Entwicklung von Modellierungskompetenzen. Wiesbaden: Springer. https://doi.org/10.1007/9783-658-27832-8.

Blomh $ø$ j, M., \& Jensen, T. H. (2003). Developing mathematical modelling competence: conceptual clarification and educational planning. Teaching Mathematics and its Applications, 22(3), 123-139. https:// doi.org/10.1093/teamat/22.3.123.

Blomhøj, M., \& Jensen, T. H. (2007). What's all the fuss about competencies? In W. Blum, P.L. Galbraith, H.-W. Henn \& M. Niss (Hrsg.), Modelling and applications in mathematics education (S. 45-56). Boston: Springer. https://doi.org/10.1007/978-0-387-29822-1_3.

Blum, W. (2002). ICMI Study 14: Applications and modelling in mathematics education - discussion document. Educational Studies in Mathematics, 51(1/2), 149-171. https://doi.org/10.1023/A: 1022435827400 .

Blum, W., \& Leiß, D. (2007). How do students and teachers deal with modelling problems? In C. Haines, P. Galbraith, W. Blum \& S. Khan (Hrsg.), Mathematical modelling (ICTMA 12): education, engineering and economics (S. 222-231). Chichester: Horwood. https://doi.org/10.1533/9780857099419.5. 221.

Blum, W., \& Schukajlow, S. (2018). Selbständiges Lernen mit Modellierungsaufgaben - Untersuchung von Lernumgebungen zum Modellieren im Projekt DISUM. In S. Schukajlow \& W. Blum (Hrsg.), Evaluierte Lernumgebungen zum Modellieren (S. 51-72). Wiesbaden: Springer. https://doi.org/10. 1007/978-3-658-20325-2_4. 
Blum, W., Neubrand, M., Ehmke, T., Senkbeil, M., Jordan, A., Ulfig, F., \& Carstensen, C. (2004). Mathematische Kompetenz. In PISA-Konsortium (Hrsg.), PISA 2003: der Bildungsstand der Jugendlichen in Deutschland. Ergebnisse des zweiten internationalen Vergleichs (S. 47-92). Münster: Waxmann.

Blum, W., \& Leiß, D. (2005). Modellieren im Unterricht mit der „Tanken“-Aufgabe. mathematik lehren, $128,18-21$.

Bond, T.G., \& Fox, C.M. (2007). Applying the Rasch model. Fundamental measurement in the human sciences. Bd. 2. New Jersey: Lawrance Erlbaum Associates.

Borromeo Ferri, R. (2006). Theoretical and empirical differentiations of phases in the modelling process. ZDM, 38(2), 86-95. https://doi.org/10.1007/BF02655883.

Borromeo Ferri, R. (2010). On the Influence of Mathematical Thinking Styles on Learners' Modelling Behavior. Journal für Mathematik-Didaktik, 31(1), 86-95.

Borromeo Ferri, R. (2018). Learning how to teach mathematical modeling in school and teacher education. Cham: Springer. https://doi.org/10.1007/978-3-319-68072-9.

Brand, S. (2014). Erwerb von Modellierungskompetenzen. Wiesbaden: Springer. https://doi.org/10.1007/ 978-3-658-06679-6.

Christou, C., Mousoulides, N., Pittalis, M., \& Pitta-Pantazi, D. (2005). Problem-solving and problem posing in a dynamic geometry environment. The Mathematics Enthusiast, 2(2), 125-143.

Djepaxhija, B., Vos, P., \& Fuglestad, A. B. (2016). Exploring grade 9 students' assumption making when mathematizing. In K. Krainer \& N. Vondrová (Hrsg.), Proceedings of the Ninth Congress of the European Society for Research in Mathematics Education. CERME 9., Prag. (S. 848-854).

Frejd, P. (2011). An investigation of mathematical modelling in the Swedish national course tests in mathematics. In Proceedings of the Seventh Congress of the European Society for Research in Mathematics Education (S. 947-956). CERME 7: Rzeszów.

Galbraith, P., \& Clatworthy, N. J. (1990). Beyond standard models?meeting the challenge of modelling. Educational Studies in Mathematics, 21(2), 137-163. https://doi.org/10.1007/BF00304899.

Galbraith, P., Renshaw, P., Goos, M., \& Geiger, V. (2003). Technology-enriched classrooms: some implications for teaching applications and modelling. In Q. Y. Jiang, Q.-X. Ye \& W. Blum (Hrsg.), Mathematical Modelling in Education and Culture - ICTMA 10 (S. 111-125). Chichester: Horwood.

Geiger, V. (2011). Factors affecting teachers' adoption of innovative practices with technology and mathematical modelling. In G. Kaiser, W. Blum, R. Borromeo Ferri \& G. Stillman (Hrsg.), Trends in teaching and learning of mathematical modelling (Bd. 1, S. 305-314). Dordrecht: Springer. https:// doi.org/10.1007/978-94-007-0910-2_31.

Greefrath, G. (2015). Problem solving methods for mathematical modelling. In G. Stillman, W. Blum \& M. Salett Biembengut (Hrsg.), Mathematical modelling in education research and practice: cultural, social and cognitive influences (S. 173-183). Cham: Springer. https://doi.org/10.1007/978-3-31918272-8_13.

Greefrath, G., \& Mühlenfeld, U. (2007). Realitätsbezogene Aufgaben für die Sekundarstufe II. Troisdorf: Bildungsverlag Eins.

Greefrath, G., \& Siller, H.-S. (2017). Modelling and simulation with the help of digital tools. In G. Stillman, W. Blum \& G. Kaiser (Hrsg.), Mathematical modelling and applications (S. 529-539). Cham: Springer. https://doi.org/10.1007/978-3-319-62968-1_44.

Greefrath, G. (2011). Using technologies: new Possibilites of teaching and learning modelling-overview: ICTMA 14. In G. Kaiser, et al. (Hrsg.), Trends in teaching and learning of mathematical modelling (S. 301-304). Dordrecht: Springer.

Greefrath, G., Hertleif, C., \& Siller, H.-S. (2018). Mathematical modelling with digital tools-a quantitative study on mathematising with dynamic geometry software. ZDM, 50(1-2), 233-244. https://doi. org/10.1007/s11858-018-0924-6.

Greefrath, G., Kaiser, G., Blum, W., \& Ferri, B. R. (2013). Mathematisches Modellieren - Eine Einführung in theoretische und didaktische Hintergründe. In R. Borromeo Ferri, G. Greefrath \& G. Kaiser (Hrsg.), Mathematisches Modellieren für Schule und Hochschule (S. 11-37). Wiesbaden: Springer. https://doi. org/10.1007/978-3-658-01580-0_1.

Haines, C., Crouch, R., \& Davis. (2001). Understanding Students' Modeling Skills. In J.F. Matos, S. K. Houston, \& W. Blum (Hrsg.), Modelling and Mathematics Education: ICTMA 9 - Applications in Science and Technology (S. 366-380). Chichester: Horwood.

Hankeln, C. (2019). Mathematisches Modellieren mit dynamischer Geometrie-Software: Ergebnisse einer Interventionsstudie. Wiesbaden: Springer. https://doi.org/10.1007/978-3-658-23339-6.

Hankeln, C., \& Beckschulte, C. (2020). Teilkompetenzen des Modellierens und ihre Erfassung - Darstellung einer Testentwicklung. In G. Greefrath \& K. Maß (Hrsg.), Modellierungskompetenzen - 
Diagnose und Bewertung (S. 65-86). Berlin, Heidelberg: Springer. https://doi.org/10.1007/978-3662-60815-9_4.

Hankeln, C., Adamek, C., \& Greefrath, G. (2019). Assessing sub-competencies of mathematical modelling-development of a new test instrument. In G. Stillman \& J. Brown (Hrsg.), Lines of inquiry in mathematical modelling research in education (S. 143-160). Cham: Springer. https://doi.org/10. 1007/978-3-030-14931-4_8.

Harks, B., Klieme, E., Hartig, J., \& Leiss, D. (2014). Separating cognitive and content domains in mathematical competence. Educational Assessment, 19, 243-266. https://doi.org/10.1080/10627197.2014. 964114.

Hartig, J. (2006). Kompetenzen als Ergebnisse von Bildungsprozessen. dipf informiert. Journal des Deutschen Instituts für internationale Pädagogische Forschung, 10, 2-7.

Hattie, J. (2010). Visible learning. London: Routledge. Reprint

Haug, R. (2008). Problemlösen Lernen mit interaktiven Lernumgebungen. Eine empirische Studie zur Förderung heuristischer Strategien durch den Einsatz Dynamischer Geometrie-Software. In E. Vásárhely (Hrsg.), Beiträge zum Mathematikunterricht (S. 449-452). Münster: WTM.

Hertleif, C. (2018). Wie groß ist die Etage? Dynamische Geometrie Software (DGS) als Hilfsmittel beim Modellieren nutzen. Mathematik lehren, 207, 16-19.

Hertleif, C., \& Adamek, C. (2016). Der Pillnitzer Schlossgarten - Modellieren in Theorie und Praxis. Mathematik, 5-10(37), 42-54.

Hertleif, C., \& Greefrath, G. (2016). Mathematisches Modellieren mit digitalen Werkzeugen - Eine Fallstudie mit Dynamischer Geometrie-Software. In Institut für Mathematik und Informatik der Pädagogischen Hoschschule Heidelberg (Hrsg.), Beiträge zum Mathematikunterricht (S. 425-428). Münster: WTM. https://doi.org/10.17877/de290r-17448.

Kaiser, G. (2007). Modelling and modelling competencies in school. In C. R. Haines, P.L. Galbraith, W. Blum \& S. Khan (Hrsg.), Mathematical modelling (ICTMA 12): education, engineering and economics (S. 110-119). Chichester: Horwood. https://doi.org/10.1533/9780857099419.3.110.

Kaiser, G., \& Sriraman, B. (2006). A global survey of international perspectives on modelling in mathematics education. ZDM, 38(3), 302-310. https://doi.org/10.1007/BF02652813.

Kaiser, G., Blum, W., Borromeo Ferri, R., \& Greefrath, G. (2015). Anwendungen und Modellieren. In R. Bruder, L. Hefendehl-Hebeker, B. Schmidt-Thieme \& H.-G. Weigand (Hrsg.), Handbuch der Mathematikdidaktik (S. 357-383). Berlin, Heidelberg: Springer. https://doi.org/10.1007/978-3-64235119-8_13.

KMK (Hrsg.). (2004). Bildungsstandards im Fach Mathematik für den Mittleren Schulabschluss. Beschluss vom 4.12.2003. Neuwied: Luchterhand.

Kreckler, J. (2015). Standortplanung und Geometrie. Wiesbaden: Springer. https://doi.org/10.1007/978-3658-09261-0.

Kuha, J. (2004). AIC and BIC. Comparisons of assumptions and performance. Sociological Methods and Research, 33(2), 188-229.

Leiss, D., \& Tropper, N. (2014). Umgang mit Heterogenität im Mathematikunterricht. Berlin, Heidelberg: Springer. https://doi.org/10.1007/978-3-642-45109-6.

Leiss, D., Schukajlow, S., Blum, W., Messner, R., \& Pekrun, R. (2010). The role of the situation model in mathematical modelling - task analyses, student competencies, and teacher interventions. Journal für Mathematik-Didaktik, 31(1), 119-141. https://doi.org/10.1007/s13138-010-0006-y.

Lindmeier, A., Ufer, S., \& Reiss, K. (2018). Modellieren lernen mit heuristischen Lösungsbeispielen. Interventionen zum selbstständigkeitsorientierten Erwerb von Modellierungskompetenzen. In S. Schukajlow \& W. Blum (Hrsg.), Evaluierte Lernumgebungen zum Modellieren (S. 265-288). Wiesbaden: Springer. https://doi.org/10.1007/978-3-658-20325-2_13.

Maßß, K. (2004). Mathematisches Modellieren im Unterricht. Ergebnisse einer empirischen Studie. Hildesheim: Franzbecker.

Maßß, K. (2006). What are modelling competencies? ZDM, 38(2), 113-142. https://doi.org/10.1007/ $\mathrm{BF} 02655885$.

Meyer (2013). GeoGebra - Aspekte einer dynamischen Geometrisoftware. In M. Ruppert \& J. Wörler (Hrsg.), Technologien im Mathematikunterricht (S. 5-12). Wiesbaden: Springer. https://doi.org/10. 1007/978-3-658-03008-7_1.

Meyer, M., \& Voigt, J. (2010). Rationale Modellierungsprozesse. In B. Brandt, M. Fetzer \& M. Schütte (Hrsg.), Auf den Spuren interpretativer Unterrichtsforschung in der Mathematikdidaktik: Götz. Krummheuer zum 60. Geburtstag (S. 117-148). Münster: Waxmann. 
Mousoulides, N. G. (2011). Geogebra as a conceptual tool for modeling real world problems. In L. Bu \& R. Schoen (Hrsg.), Model-centered learning (S. 105-118). Rotterdam: SensePublishers. https://doi. org/10.1007/978-94-6091-618-2_8.

Niss, M., Blum, W., \& Galbraith, P. (2007). Introduction. In W. Blum, P.L. Galbraith, H.-W. Henn \& M. Niss (Hrsg.), Modelling and applications in mathematics education. The 14th ICMI study (Bd. 10, S. 3-32). Boston: Springer. https://doi.org/10.1007/978-0-387-29822-1_1.

Olive, J., Makar, K., Hoyos, V., Kor, L. K., Kosheleva, O., \& Sträßer, R. (2009). Mathematical knowledge and practices resulting from access to digital technologies. In C. Hoyles \& J.-B. Lagrange (Hrsg.), Mathematics education and technology-rethinking the terrain (Bd. 13, S. 133-177). Boston: Springer. https://doi.org/10.1007/978-1-4419-0146-0_8.

Pollak, H.O. (1977). The interaction between mathematics and other school subjects (including integrated courses). In H. Athen \& H. Kunle (Hrsg.), Proceedings of the third international congress on mathematical education (S. 255-264). Karlsruhe: Zentralblatt für Didaktik der Mathematik.

Reiss, K., \& Renkl, A. (2002). Learning to prove: the idea of heuristic examples. Zentralblatt für Didaktik der Mathematik, 34(1), 29-35. https://doi.org/10.1007/BF02655690.

Reit, X.-R. (2016). Denkstrukturen in Lösungsansätzen von Modellierungsaufgaben. Wiesbaden: Springer. https://doi.org/10.1007/978-3-658-13189-0.

Rellensmann, J., Schukajlow, S., \& Leopold, C. (2017). Make a drawing. Effects of strategic knowledge, drawing accuracy, and type of drawing on students' mathematical modelling performance. Educational Studies in Mathematics, 95(1), 53-78. https://doi.org/10.1007/s10649-016-9736-1.

Reusser, K. (1997). Erwerb mathematischer Kompetenzen: Literaturüberblick. In F. E. Weinert \& A. Helmke (Hrsg.), Entwicklung im Grundschulalter (S. 141-155). Weinheim: Beltz, Psychologie-Verl.-Union.

Roeling, M. B. (2015). Konzeptuelles Wissen und Konzeptentwicklung in Biologie. Eine Längsschnittstudie. Biologie lernen und lehren. Berlin: Logos.

Rost, J. (2004). Testtheorie - Testkonstruktion. Bern: Hans Huber.

Sacristán, A. I., Calder, N., Rojano, T., Santos-Trigo, M., Friedlander, A., \& Meissner, H. (2009). The influence and shaping of digital technologies on the learning — and learning trajectories — of mathematical concepts. In C. Hoyles \& J.-B. Lagrange (Hrsg.), Mathematics education and technology-rethinking the terrain (Bd. 13, S. 179-226). Boston: Springer. https://doi.org/10.1007/978-1-4419-0146-0_9.

Savelsbergh, E., Drijvers, P., van de Giessen, C., Heck, A., Hooyman, K., Kruger, J., et al. (2008). Modelleren en computermodellen in de $\beta$-vakken. Advies aan de gezamenlijke $\beta$-vernieuwingscommissies. Utrecht: Freudenthal Instituut voor Didactiek van Wiskunde en Natuurwetenschappen.

Schukajlow, S., \& Leiss, D. (2011). Selbstberichtete Strategienutzung und mathematische Modellierungskompetenz. Journal für Mathematik-Didaktik, 32(1), 53-77. https://doi.org/10.1007/s13138-0100023-x.

Schukajlow, S., Achmetli, K., \& Rakoczy, K. (2019). Does constructing multiple solutions for real-world problems affect self-efficacy? Educational Studies in Mathematics, 100(1), 43-60. https://doi.org/10. 1007/s10649-018-9847-y.

Schukajlow, S., Kolter, J., \& Blum, W. (2015a). Scaffolding mathematical modelling with a solution plan. ZDM, 47(7), 1241-1254. https://doi.org/10.1007/s11858-015-0707-2.

Schukajlow, S., Krug, A., \& Rakoczy, K. (2015b). Effects of prompting multiple solutions for modelling problems on students' performance. Educational Studies in Mathematics, 89(3), 393-417. https://doi. org/10.1007/s10649-015-9608-0.

Siller, H.-S., \& Greefrath, G. (2010). Mathematical modelling in class regarding to technology. In V. Durand-Guerrier, S. Soury-Lavergne \& F. Arzarello (Hrsg.), Proceedings of the Sixth Congress of the European Society for Research in Mathematics Education. CERME 6, Lyon. (S. 2136-2145).

Stillman, G. (2011). Applying Metacognitive knowledge and strategies in applications and modelling tasks at secondary school. In G. Kaiser, W. Blum, R. Borromeo Ferri \& G. Stillman (Hrsg.), Trends in teaching and learning of mathematical modelling (Bd. 1, S. 165-180). Dordrecht: Springer Netherlands. https://doi.org/10.1007/978-94-007-0910-2_18.

Stillman, G., \& Galbraith, P. L. (1998). Applying mathematics with real world connections: metacognitive characteristics of secondary students. Educational Studies in Mathematics, 36(2), 157-194. https:// doi.org/10.1023/A:1003246329257.

Tanner, H., \& Jones, S. (1995). Developing Metacognitive skills in mathematical modelling-a socioconstructivist interpretation. In C. Sloyer, W. Blum \& I. Huntley (Hrsg.), Advances and perspectives in the teaching of mathematical modelling and applications (S. 61-70). 
Tropper, N. (2019). Strategisches Modellieren durch heuristische Lösungsbeispiele: Untersuchungen von Lösungsprozeduren und Strategiewissen zum mathematischen Modellierungsprozess. Wiesbaden: Springer. https://doi.org/10.1007/978-3-658-24992-2.

Vorhölter, K., Krüger, A., \& Wendt, L. (2019). Chapter 2: metacognition in mathematical modeling-an overview. In S. A. Chamberlin \& B. Sriraman (Hrsg.), Affect in mathematical modeling (S. 29-51). Cham: Springer. https://doi.org/10.1007/978-3-030-04432-9_3.

Wright, B.D. (2003). Rack and stack: time 1 vs. time 2 or pre-test vs. Post-test. Rasch Measurement Transactions, 17(1), 905-906.

Zech, F. (2002). Grundkurs Mathematikdidaktik: theoretische und praktische Anleitungen für das Lehren und Lernen von Mathematik (10. Aufl.). Weinheim Basel: Beltz.

Zöttl, L. (2010). Modellierungskompetenz fördern mit heuristischen Lösungsbeispielen. Hildesheim: Franzbecker.

Zöttl, L., Ufer, S., \& Reiss, K. (2010). Modelling with heuristic worked examples in the KOMMA learning environment. Journal für Mathematik-Didaktik, 31(1), 143-165. https://doi.org/10.1007/s13138010-0008-9.

Zöttl, L., Ufer, S., \& Reiss, K. (2011). Assessing modelling competencies using a multidimensional IRT approach. In G. Kaiser, W. Blum, R. Borromeo Ferri \& G. Stillman (Hrsg.), Trends in teaching and learning of mathematical modelling (S. 427-437). Dordrecht: Springer. https://doi.org/10.1007/97894-007-0910-2_42. 